\title{
Restricted normal cones and the method of alternating projections: theory
}

\author{
Heinz H. Bauschke, D. Russell Luke, Hung M. Phan’, and Xianfu Wang§
}

March 1, 2013

\begin{abstract}
In this paper, we introduce and develop the theory of restricted normal cones which generalize the classical Mordukhovich normal cone. We thoroughly study these objects from the viewpoint of constraint qualifications and regularity. Numerous examples are provided to illustrate the theory. This work provides the theoretical underpinning for a subsequent article in which these tools are applied to obtain a convergence analysis of the method of alternating projections for nonconvex sets.
\end{abstract}

2010 Mathematics Subject Classification: Primary 49J52; Secondary 47H09, 90C26.

Keywords: Constraint qualification, convex set, Friedrichs angle, normal cone, nonconvex set, projection operator, restricted normal cone, superregularity.

\section{Introduction and auxiliary results}

Throughout this paper, we assume that

$$
X \text { is a Euclidean space }
$$

\footnotetext{
*Mathematics, University of British Columbia, Kelowna, B.C. V1V 1V7, Canada. E-mail: heinz . bauschke@ubc . ca.

${ }^{\dagger}$ Institut für Numerische und Angewandte Mathematik, Universität Göttingen, Lotzestr. 16-18, 37083 Göttingen, Germany. E-mail: r.luke@math.uni-goettingen.de.

‡Department of Mathematics \& Statistics, University of Victoria, PO Box 3060 STN CSC, Victoria, B.C. V8W 3R4, Canada. E-mail: hphan@uvic.ca.

§Mathematics, University of British Columbia, Kelowna, B.C. V1V 1V7, Canada. E-mail: shawn. wang@ubc.ca.
} 
(i.e., finite-dimensional real Hilbert space) with inner product $\langle\cdot, \cdot\rangle$, induced norm $\|\cdot\|$, and induced metric $d$.

Let $A$ and $B$ be nonempty closed subsets of $X$. Let us assume that $A$ and $B$ are convex and that $A \cap B \neq \varnothing$. In this case, the projection operators $P_{A}$ and $P_{B}$ (a.k.a. projectors or nearest point mappings) corresponding to $A$ and $B$, respectively, are single-valued with full domain. In order to find a point in the intersection $A$ and $B$, it is very natural to simply alternate the operator $P_{A}$ and $P_{B}$ resulting in the famous method of alternating projections (MAP). Thus, given a starting point $b_{-1} \in X$, sequences $\left(a_{n}\right)_{n \in \mathbb{N}}$ and $\left(b_{n}\right)_{n \in \mathbb{N}}$ are generated as follows:

$$
(\forall n \in \mathbb{N}) \quad a_{n}:=P_{A} b_{n-1}, \quad b_{n}:=P_{B} a_{n} .
$$

In the present consistent convex setting, it is known that both sequences have a common limit in $A \cap B$. Not surprisingly, because of its elegance and usefulness, the MAP has attracted many famous mathematicians, including John von Neumann [18] and Norbert Wiener [19] and it has been independently rediscovered repeatedly.

In this article we lay the groundwork for a comprehensive analysis of the MAP when $A$ and $B$ are possibly nonconvex. We point readers interested in reviewing the rich history of this algorithm to [2], [6], [9], and the references therein. The results in this paper are crucial for the analysis of the MAP which we present in the follow-up paper [4].

A careful study of restricted normal cones and related notions is carried out in this paper. We also allow for constraint sets that are unions of superregular (or even convex) sets; in the follow-up paper [4], we recover the known optimal convergence rate for the method of alternating projections for two subspaces. In a parallel paper [3], we apply the tools developed here to the important problem of sparsity optimization with affine constraints.

The paper is organized as follows. The restricted normal cones are introduced in Section 2. Section 3 focuses on normal cones that are restricted by affine subspaces. Further examples and results are provided in Section 4 and Section 5, where we illustrate that the restricted normal cone cannot be obtained by intersections with various natural conical supersets. Section 6 and Section 7 are devoted to constraint qualifications which describe how well the sets $A$ and $B$ relate to each other. In Section 8, we discuss regularity and superregularity, notions that extend the idea of convexity, for sets and collections of sets.

In the remainder of this section, we fix notation and collect various auxiliary results that are useful later and to make the later analysis less cluttered.

\section{Notation}

The notation employed in this article is quite standard and follows largely [5], [15], [16], and [17]; these books also provide exhaustive information on variational analysis. The real numbers are $\mathbb{R}$, the integers are $\mathbb{Z}$, and $\mathbb{N}:=\{z \in \mathbb{Z} \mid z \geq 0\}$. Further, $\mathbb{R}_{+}:=\{x \in \mathbb{R} \mid x \geq 0\}, \mathbb{R}_{++}:=$ $\{x \in \mathbb{R} \mid x>0\}$ and $\mathbb{R}_{-}$and $\mathbb{R}_{--}$are defined analogously. Let $R$ and $S$ be subsets of $X$. Then the 
closure of $S$ is $\bar{S}$, the interior of $S$ is $\operatorname{int}(S)$, the boundary of $S$ is bdry $(S)$, and the smallest affine and linear subspaces containing $S$ are aff $S$ and span $S$, respectively. The linear subspace parallel to aff $S$ is $\operatorname{par} S:=(\operatorname{aff} S)-S=(\operatorname{aff} S)-s$, for every $s \in S$. The relative interior of $S, \operatorname{ri}(S)$, is the interior of $S$ relative to aff $(S)$. The negative polar cone of $S$ is $S^{\ominus}:=\{u \in X \mid \sup \langle u, S\rangle \leq 0\}$. We also set $S^{\oplus}:=-S^{\ominus}$ and $S^{\perp}:=S^{\oplus} \cap S^{\ominus}$. We also write $R \oplus S$ for $R+S:=\{r+s \mid(r, s) \in R \times S\}$ provided that $R \perp S$, i.e., $(\forall(r, s) \in R \times S)\langle r, s\rangle=0$. The identity mapping on $X$ is Id: $X \rightarrow$ $X: x \mapsto x$. We write $F: X \rightrightarrows X$, if $F$ is a mapping from $X$ to its power set, i.e., gr $F$, the graph of $F$, lies in $X \times X$. Abusing notation slightly, we will write $F(x)=y$ if $F(x)=\{y\}$. If $f: X \rightarrow$ ]$-\infty,+\infty]$, then the epigraph of $f$ is epi $f:=\{(x, \rho) \in X \times \mathbb{R} \mid f(x) \leq \rho\}$. A nonempty subset $K$ of $X$ is a cone if $\left(\forall \lambda \in \mathbb{R}_{+}\right) \lambda K:=\{\lambda k \mid k \in K\} \subseteq K$. The smallest cone containing $S$ is denoted $\operatorname{cone}(S)$; thus, cone $(S):=\mathbb{R}_{+} \cdot S:=\left\{\rho s \mid \rho \in \mathbb{R}_{+}, s \in S\right\}$ if $S \neq \varnothing$ and cone $(\varnothing):=\{0\}$. The smallest convex and closed and convex subset containing $S$ are $\operatorname{conv}(S)$ and $\overline{\operatorname{conv}}(S)$, respectively. If $z \in X$ and $\rho \in \mathbb{R}_{++}$, then ball $(z ; \rho):=\{x \in X \mid d(z, x) \leq \rho\}$ is the closed ball centered at $z$ with radius $\rho$ while sphere $(z ; \rho):=\{x \in X \mid d(z, x)=\rho\}$ is the (closed) sphere centered at $z$ with radius $\rho$. If $u$ and $v$ are in $X$, then $[u, v]:=\{(1-\lambda) u+\lambda v \mid \lambda \in[0,1]\}$ is the line segment connecting $u$ and $v$.

\section{Projections}

Since $X$ is finite-dimensional and $A$ and $B$ are closed, the convexity of $A$ and $B$ is actually not needed in order to guarantee existence of nearest points. This gives rise to set-valued projection operators which for convenience we also denote by $P_{A}$ and $P_{B}$.

Definition 1.1 (distance and projection) Let $A$ be a nonempty subset of $X$. Then

$$
d_{A}: X \rightarrow \mathbb{R}: x \mapsto \inf _{a \in A} d(x, a)
$$

is the distance function of the set $A$ and

$$
P_{A}: X \rightrightarrows X: x \mapsto\left\{a \in A \mid d_{A}(x)=d(x, a)\right\}
$$

is the corresponding projection.

Proposition 1.2 (existence) Let $A$ be a nonempty closed subset of $X$. Then $(\forall x \in X) P_{A}(x) \neq \varnothing$.

Proof. Let $z \in X$. The function $f: X \rightarrow \mathbb{R}: x \mapsto\|x-z\|^{2}$ is continuous and $\lim _{\|x\| \rightarrow+\infty} f(x)=+\infty$. Let $\left(x_{n}\right)_{n \in \mathbb{N}}$ be a sequence in $A$ such that $f\left(x_{n}\right) \rightarrow \inf f(A)$. Then $\left(x_{n}\right)_{n \in \mathbb{N}}$ is bounded. Since $A$ is closed and $f$ is continuous, every cluster point of $\left(x_{n}\right)_{n \in \mathbb{N}}$ is a minimizer of $f$ over the set $A$, i.e., an element in $P_{A} z$.

The following result is well known.

Fact 1.3 (projection onto closed convex set) Let $C$ be a nonempty closed convex subset of $X$, and let $x$, $y$ and $p$ be in $X$. Then the following hold: 
(i) $P_{C}(x)$ is a singleton.

(ii) $P_{C}(x)=p$ if and only if $p \in C$ and $\sup \langle C-p, x-p\rangle \leq 0$.

(iii) $\left\|P_{C}(x)-P_{C}(y)\right\|^{2}+\left\|\left(\operatorname{Id}-P_{C}\right)(x)-\left(\operatorname{Id}-P_{C}\right)(y)\right\|^{2} \leq\|x-y\|^{2}$.

(iv) $\left\|P_{C}(x)-P_{C}(y)\right\| \leq\|x-y\|$.

Proof. (i)\&(ii): [2, Theorem 3.14]. (iii): [2, Proposition 4.8]. (iv): Clear from (iii).

Example 1.4 (sphere) Let $z \in X$ and $\rho \in \mathbb{R}_{++}$. Set $S:=\operatorname{sphere}(z ; \rho)$. Then

$$
(\forall x \in X) \quad P_{S}(x)= \begin{cases}z+\rho \frac{x-z}{\|x-z\|}, & \text { if } x \neq z ; \\ S, & \text { otherwise }\end{cases}
$$

Proof. Let $x \in X$. The formula is clear when $x=z$, so we assume $x \neq z$. Set

$$
c:=z+\rho \frac{x-z}{\|x-z\|} \in S
$$

and let $s=z+\rho b \in S \backslash\{c\}$, i.e., $\|b\|=1$ and $b \neq(x-z) /\|x-z\|$. Hence, using that $\|u\|-$ $\|v\| \mid\|u-v\| \Leftrightarrow\langle u, v\rangle<\|u\|\|v\|$ and because of Cauchy-Schwarz, we obtain

$$
\begin{aligned}
\|x-c\| & =|\|x-z\|-\rho|=|\|x-z\|-\|\rho b\||=|\|x-z\|-\|s-z\|| \\
& <\|x-s\| .
\end{aligned}
$$

We have thus established (5).

\section{Miscellany}

Lemma 1.5 Let $A$ and $B$ be subsets of $X$, and let $K$ be a cone in $X$. Then the following hold:

(i) cone $(A \cap B) \subseteq$ cone $A \cap$ cone $B$.

(ii) cone $(K \cap B)=K \cap$ cone $B$.

Proof. (i): Clear. (ii): By (i), cone $(K \cap B) \subseteq$ (cone $K) \cap$ (cone $B)=K \cap$ cone $B$. Now assume that $x \in(K \cap$ cone $B) \backslash\{0\}$. Then there exists $\beta>0$ such that $x / \beta \in B$. Since $K$ is a cone, $x / \beta \in K$. Thus $x / \beta \in K \cap B$ and therefore $x \in \operatorname{cone}(K \cap B)$.

Note that the inclusion in Lemma 1.5(i) may be strict: indeed, consider the case when $X=\mathbb{R}$, $A:=\{1\}$, and $B:=\{2\}$.

Lemma 1.6 (Bunt-Motzkin characterization of convexity) Let A be a nonempty closed subset of X. Then the following are equivalent: 
(i) $A$ is convex.

(ii) $P_{A}^{-1}(a)-a$ is a cone, for every $a \in A$.

(iii) $P_{A}(x)$ is a singleton, for every $x \in X$.

Proof. "(i) $\Rightarrow$ (ii)": Indeed, it is well known in convex analysis (see, e.g., [17, Proposition 6.17]) that for every $a \in A, P_{A}^{-1}(a)-a$ is equal to the normal cone (in the sense of convex analysis) of $A$ at $a$.

"(ii) $\Rightarrow$ (iii)": Let $x \in X$. By Proposition 1.2, $P_{A} x \neq \varnothing$. Take $a_{1}$ and $a_{2}$ in $P_{A} x$. Then $\left\|x-a_{1}\right\|=$ $\left\|x-a_{2}\right\|$ and $x-a_{1} \in P_{A}^{-1} a_{1}-a_{1}$. Since $P_{A}^{-1} a-a$ is a cone, we have $2\left(x-a_{1}\right) \in P_{A}^{-1} a_{1}-a_{1}$. Hence $y:=2 x-a_{1} \in P_{A}^{-1} a_{1}$ and $y-x=x-a_{1}$. Thus,

$$
\begin{aligned}
\left\langle y-a_{2}, a_{1}-a_{2}\right\rangle & =\left\langle(y-x)+\left(x-a_{2}\right),\left(a_{1}-x\right)+\left(x-a_{2}\right)\right\rangle \\
& =\left\langle y-x, a_{1}-x\right\rangle+\left\langle y-x, x-a_{2}\right\rangle+\left\langle x-a_{2}, a_{1}-x\right\rangle+\left\|x-a_{2}\right\|^{2} \\
& =\left\langle x-a_{1}, a_{1}-x\right\rangle+\left\langle x-a_{1}, x-a_{2}\right\rangle+\left\langle x-a_{2}, a_{1}-x\right\rangle+\left\|x-a_{2}\right\|^{2} \\
& =-\left\|x-a_{1}\right\|^{2}+\left\|x-a_{2}\right\|^{2} \\
& =0 .
\end{aligned}
$$

Since $a_{1} \in P_{A} y$, it follows that

$$
\begin{aligned}
\left\|y-a_{1}\right\|^{2} & =\left\|y-a_{2}\right\|^{2}+2\left\langle y-a_{2}, a_{2}-a_{1}\right\rangle+\left\|a_{1}-a_{2}\right\|^{2} \\
& =\left\|y-a_{2}\right\|^{2}+\left\|a_{1}-a_{2}\right\|^{2} \\
& \geq\left\|y-a_{2}\right\|^{2} \\
& \geq\left\|y-a_{1}\right\|^{2} .
\end{aligned}
$$

Hence equality holds throughout (9). Therefore, $a_{1}=a_{2}$.

"(iii) $\Rightarrow($ i)": This classical result due to Bunt and to Motzkin on the convexity of Chebyshev sets is well known; for proofs, see, e.g., [9, Chapter 12] or [2, Corollary 21.13].

Proposition 1.7 Let $S$ be a convex set. Then the following are equivalent.

(i) $0 \in \operatorname{ri} S$.

(ii) cone $S=\operatorname{span} S$.

(iii) $\overline{\text { cone } S}=\operatorname{span} S$.

Proof. Set $Y=\operatorname{span} S$. Then (i) $\Leftrightarrow 0$ belongs to the interior of $S$ relative to $Y$.

"(i) $\Rightarrow($ ii)": There exists $\delta>0$ such that for every $y \in Y \backslash\{0\}, \delta y /\|y\| \in S$. Hence $y \in$ cone $S$.

“(ii) $\Rightarrow(\mathrm{i}) ":$ For every $y \in Y$, there exists $\delta>0$ such that $\delta y \in S$. Now [16, Corollary 6.4.1] applies in $Y$. 
"(ii) $\Leftrightarrow($ iii)": Set $K=$ cone $S$, which is convex. By [16, Corollary 6.3.1], we have ri $K=\operatorname{ri} Y \Leftrightarrow$ $\bar{K}=\bar{Y} \Leftrightarrow \operatorname{ri} Y \subseteq K \subseteq \bar{Y}$. Since ri $Y=Y=\bar{Y}$, we obtain the equivalences: $\operatorname{ri} K=Y \Leftrightarrow \bar{K}=Y \Leftrightarrow$ $K=Y$.

\section{Restricted normal cones: basic properties}

Normal cones are fundamental objects in variational analysis; they may be used to construct subdifferential operators, and they have found many applications in optimization, optimal control, nonlinear analysis, convex analysis, etc.; see, e.g., [2], [5], [7], [14], [15], [16], [17]. One of the key building blocks is the Mordukhovich (or limiting) normal cone $N_{A}$, which is obtained by limits of proximal normal vectors. In this section, we propose a new, very flexible, normal cone of $A$, denoted by $N_{A}^{B}$, by constraining the proximal normal vectors to a set $B$.

Definition 2.1 (normal cones) Let $A$ and $B$ be nonempty subsets of $X$, and let $a$ and $u$ be in $X$. If $a \in A$, then various normal cones of $A$ at a are defined as follows:

(i) The B-restricted proximal normal cone of $A$ at $a$ is

$$
\widehat{N}_{A}^{B}(a):=\text { cone }\left(\left(B \cap P_{A}^{-1} a\right)-a\right)=\text { cone }\left((B-a) \cap\left(P_{A}^{-1} a-a\right)\right) .
$$

(ii) The (classical) proximal normal cone of $A$ at $a$ is

$$
N_{A}^{\text {prox }}(a):=\widehat{N}_{A}^{X}(a)=\text { cone }\left(P_{A}^{-1} a-a\right) .
$$

(iii) The B-restricted normal cone $N_{A}^{B}(a)$ is implicitly defined by $u \in N_{A}^{B}(a)$ if and only if there exist sequences $\left(a_{n}\right)_{n \in \mathbb{N}}$ in $A$ and $\left(u_{n}\right)_{n \in \mathbb{N}}$ in $\widehat{N}_{A}^{B}\left(a_{n}\right)$ such that $a_{n} \rightarrow a$ and $u_{n} \rightarrow u$.

(iv) The Fréchet normal cone $N_{A}^{\text {Fré }}(a)$ is implicitly defined by $u \in N_{A}^{\text {Fré }}(a)$ if and only if $(\forall \varepsilon>0)$ $(\exists \delta>0)(\forall x \in A \cap \operatorname{ball}(a ; \delta))\langle u, x-a\rangle \leq \varepsilon\|x-a\|$.

(v) The normal convex from convex analysis $N_{A}^{\text {conv }}(a)$ is implicitly defined by $u \in N_{A}^{\text {conv }}(a)$ if and only if $\sup \langle u, A-a\rangle \leq 0$.

(vi) The Mordukhovich normal cone $N_{A}(a)$ of $A$ at a is implicitly defined by $u \in N_{A}(a)$ if and only if there exist sequences $\left(a_{n}\right)_{n \in \mathbb{N}}$ in $A$ and $\left(u_{n}\right)_{n \in \mathbb{N}}$ in $N_{A}^{\text {prox }}\left(a_{n}\right)$ such that $a_{n} \rightarrow a$ and $u_{n} \rightarrow u$.

If $a \notin A$, then all normal cones are defined to be empty. 


\section{The proximal normal cone}

\author{
The restricted \\ proximal formal cone
}

Remark 2.2 Some comments regarding Definition 2.1 are in order.

(i) Clearly, the restricted proximal normal cone generalizes the notion of the classical proximal normal cone. The name "restricted" stems from the fact that the pre-image $P_{A}^{-1} a$ is restricted to the set $B$.

(ii) See [17, Example 6.16] and [15, Subsection 2.5.2.D on page 240] for further information regarding the classical proximal normal cone, including the fact that

$$
u \in N_{A}^{\text {prox }}(a) \quad \Leftrightarrow \quad a \in A \text { and }(\exists \delta>0)(\forall x \in A) \quad\langle u, x-a\rangle \leq \delta\|x-a\|^{2} .
$$

This also implies that: $N_{A}^{\text {prox }}(a)+(A-a)^{\ominus} \subseteq N_{A}^{\text {prox }}(a)$.

(iii) Note that $\operatorname{gr} N_{A}^{B}=(A \times X) \cap \overline{\operatorname{gr} \widehat{N}_{A}^{B}}$. Put differently, $N_{A}^{B}(a)$ is the outer (or upper Kuratowski) limit of $\widehat{N}_{A}^{B}(x)$ as $x \rightarrow a$ in $A$, written

$$
N_{A}^{B}(a)=\varlimsup_{\substack{x \rightarrow a \\ x \in A}} \widehat{N}_{A}^{B}(x) .
$$

See also [17, Chapter 4].

(iv) See [15, Definition 1.1] or [17, Definition 6.3] (where this is called the regular normal cone) for further information regarding $N_{A}^{\text {Fré }}(a)$.

(v) The Mordukhovich normal cone is also known as the basic or limiting normal cone. Note that $N_{A}=N_{A}^{X}$ and $\operatorname{gr} N_{A}=(A \times X) \cap \overline{\operatorname{gr} \widehat{N}_{A}^{X}}=(A \times X) \cap \overline{\operatorname{gr} N_{A}^{\text {prox }}}$ and once again $N_{A}(a)$ is the outer (or upper Kuratowski) limit of $\widehat{N}_{A}^{X}(x)$ or $N_{A}^{\text {prox }}(x)$ as $x \rightarrow a$ in $A$. See also [15, page 141] for historical notes.

The next result presents useful characterizations of the Mordukhovich normal cone.

Proposition 2.3 (characterizations of the Mordukhovich normal cone) Let $A$ be a nonempty closed subset of $X$, let $a \in A$, and let $u \in X$. Then the following are equivalent: 
(i) $u \in N_{A}(a)$.

(ii) There exist sequences $\left(\lambda_{n}\right)_{n \in \mathbb{N}}$ in $\mathbb{R}_{+},\left(b_{n}\right)_{n \in \mathbb{N}}$ in $X,\left(a_{n}\right)_{n \in \mathbb{N}}$ in $A$ such that $a_{n} \rightarrow a, \lambda_{n}\left(b_{n}-\right.$ $\left.a_{n}\right) \rightarrow u$, and $(\forall n \in \mathbb{N}) a_{n} \in P_{A} b_{n}$.

(iii) There exist sequences $\left(\lambda_{n}\right)_{n \in \mathbb{N}}$ in $\mathbb{R}_{+},\left(x_{n}\right)_{n \in \mathbb{N}}$ in $X,\left(a_{n}\right)_{n \in \mathbb{N}}$ in $A$ such that $x_{n} \rightarrow a, \lambda_{n}\left(x_{n}-\right.$ $\left.a_{n}\right) \rightarrow u$, and $(\forall n \in \mathbb{N}) a_{n} \in P_{A} x_{n}$. (This also implies $\left.a_{n} \rightarrow a_{\text {.) }}\right)$

(iv) There exist sequences $\left(a_{n}\right)_{n \in \mathbb{N}}$ in $A$ and $\left(u_{n}\right)_{n \in \mathbb{N}}$ in $X$ such that $a_{n} \rightarrow a, u_{n} \rightarrow u$, and $(\forall n \in \mathbb{N})$ $u_{n} \in N_{A}^{\mathrm{Fré}}\left(a_{n}\right)$.

Proof. “(i) $\Leftrightarrow($ ii)": Clear from Definition 2.1(vi).

"(iii) $\Leftrightarrow\left(\right.$ iv)": Noting that the definition of $N_{A}(a)$ in [15] is the one given in (iv), we see that this equivalence follows from [15, Theorem 1.6].

"(ii) $\Rightarrow$ (iii)": Let $\left(\lambda_{n}\right)_{n \in \mathbb{N}},\left(a_{n}\right)_{n \in \mathbb{N}}$, and $\left(b_{n}\right)_{n \in \mathbb{N}}$ be as in (ii). For every $n \in \mathbb{N}$, since $a_{n} \in P_{A} b_{n}$, [17, Example 6.16] implies that $a_{n} \in P_{A}\left[a_{n}, b_{n}\right]$. Now let $\left(\varepsilon_{n}\right)_{n \in \mathbb{N}}$ be a sequence in $] 0,1[$ such that $\varepsilon_{n} a_{n} \rightarrow 0$ and $\varepsilon_{n} b_{n} \rightarrow 0$. Set

$$
(\forall n \in \mathbb{N}) \quad x_{n}=\left(1-\varepsilon_{n}\right) a_{n}+\varepsilon_{n} b_{n}=a_{n}+\varepsilon_{n}\left(b_{n}-a_{n}\right) \in\left[a_{n}, b_{n}\right] .
$$

Then $x_{n} \rightarrow a$ and $(\forall n \in \mathbb{N}) a_{n} \in P_{A} x_{n}$. Furthermore, $\left(\lambda_{n} / \varepsilon_{n}\right)_{n \in \mathbb{N}}$ lies in $\mathbb{R}_{+}$and

$$
\left(\lambda_{n} / \varepsilon_{n}\right)\left(x_{n}-a_{n}\right)=\lambda_{n}\left(b_{n}-a_{n}\right) \rightarrow u .
$$

"(iii) $\Rightarrow$ (ii)": Let $\left(\lambda_{n}\right)_{n \in \mathbb{N}},\left(x_{n}\right)_{n \in \mathbb{N}}$, and $\left(a_{n}\right)_{n \in \mathbb{N}}$ be as in (iii). Since $x_{n} \rightarrow a$ and $a \in A$, we deduce that $0 \leq\left\|x_{n}-a_{n}\right\|=d_{A}\left(x_{n}\right) \leq\left\|x_{n}-a\right\| \rightarrow 0$. Hence $x_{n}-a_{n} \rightarrow 0$ which implies that $a_{n}-a=a_{n}-x_{n}+x_{n}-a \rightarrow 0+0=0$. Therefore, (ii) holds with $\left(b_{n}\right)_{n \in \mathbb{N}}=\left(x_{n}\right)_{n \in \mathbb{N}}$.

Here are some basic properties of the restricted normal cone and its relation to various classical cones.

Lemma 2.4 (basic inclusions among the normal cones) Let $A$ and $B$ be nonempty subsets of $X$, and let $a \in A$. Then the following hold:

(i) $N_{A}^{\text {conv }}(a) \subseteq N_{A}^{\text {prox }}(a)$.

(ii) $\widehat{N}_{A}^{B}(a)=\operatorname{cone}\left((B-a) \cap\left(P_{A}^{-1} a-a\right)\right) \subseteq(\operatorname{cone}(B-a)) \cap N_{A}^{\text {prox }}(a)$.

(iii) $\widehat{N}_{A}^{B}(a) \subseteq \widehat{N}_{A}^{X}(a)=N_{A}^{\text {prox }}(a)$ and $N_{A}^{B}(a) \subseteq N_{A}(a)$.

(iv) $\widehat{N}_{A}^{B}(a) \subseteq N_{A}^{B}(a)$.

(v) If $A$ is closed, then $N_{A}^{\text {prox }}(a) \subseteq N_{A}^{\text {Fré }}(a)$.

(vi) If $A$ is closed, then $N_{A}^{\text {Fré }}(a) \subseteq N_{A}(a)$. 
(vii) If $A$ is closed and convex, then $\widehat{N}_{A}^{X}(a)=N_{A}^{\text {prox }}(a)=N_{A}^{\text {Fré }}(a)=N_{A}^{\text {conv }}(a)=N_{A}(a)$.

(viii) If $a \in \operatorname{ri}(A)$, then $\widehat{N}_{A}^{\text {aff }(A)}(a)=N_{A}^{\text {aff }(A)}(a)=\{0\}$.

(ix) $(\operatorname{aff}(A)-a)^{\perp} \subseteq(A-a)^{\ominus}$.

(x) $(A-a)^{\ominus} \cap \operatorname{cone}(B-a) \subseteq \widehat{N}_{A}^{B}(a) \subseteq$ cone $(B-a)$.

Proof. (i): Take $u \in N_{A}^{\text {conv }}(a)$ and fix an arbitrary $\delta>0$. Then $(\forall x \in A)\langle u, x-a\rangle \leq 0 \leq \delta\|x-a\|^{2}$. In view of (12), $u \in N_{A}^{\text {prox }}(a)$.

(ii): In view of Lemma 1.5, the definitions yield

$$
\begin{aligned}
\widehat{N}_{A}^{B}(a) & =\text { cone }\left(\left(B \cap P_{A}^{-1} a\right)-a\right)=\text { cone }\left((B-a) \cap\left(P_{A}^{-1} a-a\right)\right) \\
& \subseteq \text { cone }\left((B-a) \cap \operatorname{cone}\left(P_{A}^{-1} a-a\right)\right)=\text { cone }\left((B-a) \cap N_{A}^{\text {prox }}(a)\right) \\
& =\operatorname{cone}(B-a) \cap N_{A}^{\text {prox }}(a) .
\end{aligned}
$$

(iii), (iv) and (ix): This is obvious.

(v): Assume that $A$ is closed and take $u \in N_{A}^{\text {prox }}(a)$. By (12), there exists $\rho>0$ such that $(\forall x \in A)\langle u, x-a\rangle \leq \rho\|x-a\|^{2}$. Now let $\varepsilon>0$ and set $\delta=\varepsilon / \rho$. If $x \in A \cap \operatorname{ball}(a ; \delta)$, then $\langle u, x-a\rangle \leq \rho\|x-a\|^{2} \leq \rho \delta\|x-a\|=\varepsilon\|x-a\|$. Thus, $u \in N_{A}^{\text {Fré }}(a)$.

(vi): This follows from Proposition 2.3.

(vii): Since $A$ is closed, it follows from (i), (v), and (vi) that

$$
N_{A}^{\text {conv }}(a) \subseteq N_{A}^{\text {prox }}(a) \subseteq N_{A}^{\text {Fré }}(a) \subseteq N_{A}(a) .
$$

On the other hand, by [15, Proposition 1.5], $N_{A}(a) \subseteq N_{A}^{\text {conv }}(a)$ because $A$ is convex.

(viii): By assumption, $(\exists \delta>0) \operatorname{ball}(a ; \delta) \cap \operatorname{aff}(A) \subseteq A$. Hence $\operatorname{aff}(A) \cap P_{A}^{-1} a=\{a\}$ and thus $\widehat{N}_{A}^{\text {aff }(A)}(a)=\{0\}$. Since $a \in \operatorname{ri}(A)$, it follows that $(\forall x \in \operatorname{ball}(a ; \delta / 2) \cap \operatorname{aff}(A)) \widehat{N}_{A}^{\text {aff }(A)}(x)=\{0\}$. Therefore, $N_{A}^{\text {aff }(A)}(a)=\{0\}$.

(x): Take $u \in\left((A-a)^{\ominus} \cap \operatorname{cone}(B-a)\right) \backslash\{0\}$, say $u=\lambda(b-a)$, where $b \in B$ and $\lambda>0$. Then $0 \geq \sup \langle A-a, u\rangle=\lambda \sup \langle A-a, b-a\rangle=\sup \lambda\langle\overline{\operatorname{conv}} A-a, b-a\rangle$. By Fact 1.3(ii), $a=P_{\text {conv } A} b$ and hence $a=P_{A} b$. It follows that $u \in \operatorname{cone}\left(\left(B \cap P_{A}^{-1} a\right)-a\right)$. The left inclusion thus holds. The right inclusion is clear.

Remark 2.5 (on closedness of normal cones) Let $A$ be a nonempty subset of $X$, let $a \in A$, and let $B$ be a subset of $X$. Then $N_{A}^{B}(a), N_{A}(a)$, and $N_{A}^{\text {conv }}(a)$ are obviously closed-this is also true for $N_{A}^{\text {Fré }}(a)$ but requires some work (see [17, Proposition 6.5]). On the other hand, the classical proximal normal cone $N_{A}^{\text {prox }}(a)=\widehat{N}_{A}^{X}(a)$ is not necessarily closed (see, e.g., [17, page 213]), and hence neither is $\widehat{N}_{A}^{B}(a)$. For a concrete example, suppose that $X=\mathbb{R}^{2}$, that $A=\{(0,0)\}$, that $B=\mathbb{R} \times\{1\}$ and that $a=(0,0)$. Then $\widehat{N}_{A}^{B}(a)=\left(\mathbb{R} \times \mathbb{R}_{++}\right) \cup\{(0,0)\}$, which is not closed; however, the classical proximal normal cone $N_{A}^{\text {prox }}(a)=\mathbb{R}^{2}$ is closed. 
The sphere is a nonconvex set for which all classical normal cones coincide:

Example 2.6 (classical normal cones of the sphere) Let $z \in X$ and $\rho \in \mathbb{R}_{++}$. Set $S:=\operatorname{sphere}(z ; \rho)$ and let $s \in S$. Then $N_{S}^{\text {prox }}(s)=\widehat{N}_{S}^{X}(s)=N_{S}^{\text {Fré }}(s)=N_{S}(s)=\mathbb{R}(s-z)$.

Proof. By Example 1.4, we have $P_{S}^{-1}(s)=z+\mathbb{R}_{+}(s-z)$ and so $P_{S}^{-1}(s)-s=[-1,+\infty[\cdot(s-z)$. Hence, using Lemma 2.4(v)\&(vi), we have

$$
\begin{aligned}
N_{S}^{\text {prox }}(s) & =\widehat{N}_{S}^{X}(s)=\mathbb{R}(s-z) \subseteq N_{S}^{\text {Fré }}(s) \subseteq N_{S}(s) \\
& =\varlimsup_{\substack{s^{\prime} \rightarrow s \\
s^{\prime} \in S}} N_{S}^{\text {prox }}\left(s^{\prime}\right)=\varlimsup_{\substack{s^{\prime} \rightarrow s \\
s^{\prime} \in S}} \mathbb{R}\left(s^{\prime}-z\right)=\mathbb{R}(s-z) \\
& =N_{S}^{\text {prox }}(s),
\end{aligned}
$$

as announced.

Here are some elementary yet useful calculus rules.

Proposition 2.7 Let $A, A_{1}, A_{2}, B, B_{1}$, and $B_{2}$ be nonempty subsets of $X$, let $c \in X$, and suppose that $a \in A \cap A_{1} \cap A_{2}$. Then the following hold:

(i) If $A$ and $B$ are convex, then $\widehat{N}_{A}^{B}(a)$ is convex.

(ii) $\widehat{N}_{A}^{B_{1} \cup B_{2}}(a)=\widehat{N}_{A}^{B_{1}}(a) \cup \widehat{N}_{A}^{B_{2}}(a)$ and $N_{A}^{B_{1} \cup B_{2}}(a)=N_{A}^{B_{1}}(a) \cup N_{A}^{B_{2}}(a)$.

(iii) If $B \subseteq A$, then $\widehat{N}_{A}^{B}(a)=N_{A}^{B}(a)=\{0\}$.

(iv) If $A_{1} \subseteq A_{2}$, then $\widehat{N}_{A_{2}}^{B}(a) \subseteq \widehat{N}_{A_{1}}^{B}(a)$.

(v) $-\widehat{N}_{A}^{B}(a)=\widehat{N}_{-A}^{-B}(-a),-N_{A}^{B}(a)=N_{-A}^{-B}(-a)$, and $-N_{A}(a)=N_{-A}(-a)$.

(vi) $\widehat{N}_{A}^{B}(a)=\widehat{N}_{A-c}^{B-c}(a-c)$ and $N_{A}^{B}(a)=N_{A-c}^{B-c}(a-c)$.

Proof. It suffices to establish the conclusions for the restricted proximal normal cones since the restricted normal cone results follows by taking closures (or outer limits). (i): We assume that $B \cap P_{A}^{-1} a \neq \varnothing$, for otherwise the conclusion is clear. Then $P_{A}^{-1}(a)=P_{\bar{A}}^{-1} a=\left(\operatorname{Id}+N_{\bar{A}}\right) a$ is convex (as the image of the maximally monotone operator $\operatorname{Id}+N_{\bar{A}}$ at $\left.a\right)$. Hence $\left(B \cap P_{A}^{-1} a\right)-a$ is convex as well, and so is its conical hull, which is $\widehat{N}_{A}^{B}(a)$. (ii): Since $\left(\left(B_{1} \cup B_{2}\right) \cap P_{A}^{-1} a\right)-a=\left(\left(B_{1} \cap\right.\right.$ $\left.\left.P_{A}^{-1} a\right)-a\right) \cup\left(\left(B_{2} \cap P_{A}^{-1} a\right)-a\right)$, the result follows by taking the conical hull. (iii): Clear, because $\left(B \cap P_{A}^{-1} a\right)-a$ is either empty or equal to $\{0\}$. (iv): Suppose $\lambda(b-a) \in \widehat{N}_{A_{2}}^{B}(a)$, where $\lambda \geq 0$, $b \in B$, and $a \in P_{A_{2}} b$. Since $a \in A_{1} \subseteq A_{2}$, we have $a \in P_{A_{1}} b$. Hence $\lambda(b-a) \in \widehat{N}_{A_{1}}^{B}(a)$. (v): This follows by using elementary manipulations and the fact that $P_{-A}=(-\mathrm{Id}) \circ P_{A} \circ(-\mathrm{Id})$. (vi): This follows readily from the fact that $P_{A-c}^{-1}(a-c)=P_{A}^{-1}(a)-c$.

Remark 2.8 The restricted normal cone counterparts of items (i) and (iv) are false in general; see Example 4.1 (and also Example 4.4(iv)) below. 
The Mordukhovich normal cone (and hence also the Clarke normal cone which contains the Mordukhovich normal cone) strictly contains $\{0\}$ at boundary points (see [15, Corollary 2.24] or [17, Exercise 6.19]); however, the restricted normal cone can be $\{0\}$ at boundary points as we illustrate next.

Example 2.9 (restricted normal cone at boundary points) Suppose that $X=\mathbb{R}^{2}$, set $A:=$ ball $(0 ; 1)=\left\{x \in \mathbb{R}^{2} \mid\|x\| \leq 1\right\}$ and $B:=\mathbb{R} \times\{2\}$, and let $a=\left(a_{1}, a_{2}\right) \in A$. Then

$$
\widehat{N}_{A}^{B}(a)= \begin{cases}\mathbb{R}_{+} a, & \text { if }\|a\|=1 \text { and } a_{2}>0 ; \\ \{(0,0)\}, & \text { otherwise. }\end{cases}
$$

Consequently,

$$
N_{A}^{B}(a)= \begin{cases}\mathbb{R}_{+} a, & \text { if }\|a\|=1 \text { and } a_{2} \geq 0 ; \\ \{(0,0)\}, & \text { otherwise. }\end{cases}
$$

Thus the restricted normal cone is $\{(0,0)\}$ for all boundary points in the lower half disk that do not "face" the set $B$.

Remark 2.10 In contrast to Example 2.9, we shall see in Corollary 3.11(ii) below that if $A$ is closed, $B$ is the affine hull of $A$, and $a$ belongs to the relative boundary of $A$, then the restricted normal cone $N_{A}^{B}(a)$ strictly contains $\{0\}$.

\section{Restricted normal cones and affine subspaces}

In this section, we consider the case when the restricting set is a suitable affine subspace. This results in further calculus rules and a characterization of interiority notions.

The following four lemmas are useful in the derivation of the main results in this section.

Lemma 3.1 Let $A$ and $B$ be nonempty subsets of $X$, and suppose that $c \in A \cap B$. Then

$$
\operatorname{aff}(A \cup B)-c=\operatorname{span}(B-A) .
$$

Proof. Since $c \in A \cap B \subseteq A \cup B$, it is clear that the $\operatorname{aff}(A \cup B)-c$ is a subspace. On the one hand, if $a \in A$ and $b \in B$, then $b-a=1 \cdot b+(-1) \cdot a+1 \cdot c-c \in \operatorname{aff}(A \cup B)-c$. Hence $B-A \subseteq \operatorname{aff}(A \cup B)-c$ and thus $\operatorname{span}(B-A) \subseteq \operatorname{aff}(A \cup B)-c$. On the other hand, if $x \in$ $\operatorname{aff}(A \cup B)$, say $x=\sum_{i \in I} \lambda_{i} a_{i}+\sum_{j \in J} \mu_{j} b_{j}$, where each $a_{i}$ belongs to $A$, each $b_{j}$ belongs to $B$, and $\sum_{i \in I} \lambda_{i}+\sum_{j \in J} \mu_{j}=1$, then $x-c=\sum_{i \in I}\left(-\lambda_{i}\right)\left(c-a_{i}\right)+\sum_{j \in I} \mu_{j}\left(b_{j}-c\right) \in \operatorname{span}(B-A)$. Thus $\operatorname{aff}(A \cup B)-c \subseteq \operatorname{span}(B-A)$.

Lemma 3.2 Let $A$ be a nonempty subset of $X$, let $a \in A$, and let $u \in(\operatorname{aff}(A)-a)^{\perp}$. Then

$$
(\forall x \in X) \quad P_{A}(x+u)=P_{A}(x) .
$$


Proof. Let $x \in X$. For every $b \in A$, we have

$$
\begin{aligned}
\|u+x-b\|^{2} & =\|u\|^{2}+2\langle u, x-b\rangle+\|x-b\|^{2} \\
& =\|u\|^{2}+2\langle u, x-a\rangle+2\langle u, a-b\rangle+\|x-b\|^{2} \\
& =\|u\|^{2}+2\langle u, x-a\rangle+\|x-b\|^{2} .
\end{aligned}
$$

Hence $P_{A}(x+u)=\operatorname{argmin}_{b \in A}\|u+x-b\|^{2}=\operatorname{argmin}_{b \in A}\|x-b\|^{2}=P_{A} x$, as announced.

Lemma 3.3 Let $A$ be a nonempty subset of $X$, and let $L$ be an affine subspace of $X$ containing $A$. Then

$$
P_{A}=P_{A} \circ P_{L}
$$

Proof. Let $a \in A$ and $x \in X$, and set $b=P_{L} x$. Using [2, Corollary 3.20(i)], we have $x-b \in$ $(L-a)^{\perp} \subset(\operatorname{aff}(A)-a)^{\perp}$. In view of Lemma 3.2, we deduce that $\left(P_{A} \circ P_{L}\right) x=P_{A}(b)=P_{A}(b+$ $(x-b))=P_{A} x$.

Lemma 3.4 Let $A$ be a nonempty subset of $X$, let $a \in A$, and let $L$ be an affine subspace of $X$ containing $A$. Then the following hold:

(i) $\widehat{N}_{A}^{L}(a) \perp(L-a)^{\perp}$.

(ii) $N_{A}^{L}(a) \perp(L-a)^{\perp}$.

Proof. Observe that $L-a=\operatorname{par}(A)$ does not depend on the concrete choice of $a \in A$. (i): Using Lemma 2.4(x), we see that $\widehat{N}_{A}^{L}(a) \subseteq \operatorname{cone}(L-a) \subseteq \operatorname{span}(L-a) \perp(\operatorname{span}(L-a))^{\perp}=(L-a)^{\perp}=$ $(\operatorname{par} A)^{\perp}$. (ii): By (i), $\operatorname{ran} \widehat{N}_{A}^{L} \subseteq \operatorname{par} A$. Since $\operatorname{ran} N_{A}^{L} \subseteq \overline{\operatorname{ran} \widehat{N}_{A}^{L}}$, it follows that $\operatorname{ran} N_{A}^{L} \subseteq \operatorname{par} A=$ $L-a$.

For a normal cone restricted to certain affine subspaces, it is possible to derive precise relationships to the Mordukhovich normal cone.

Theorem 3.5 (restricted vs Mordukhovich normal cone) Let $A$ and $B$ be nonempty subsets of $X$, suppose that $a \in A$, and let $L$ be an affine subspace of $X$ containing $A$. Then the following hold:

$$
\begin{aligned}
& \widehat{N}_{A}^{X}(a)=\widehat{N}_{A}^{L}(a) \oplus(L-a)^{\perp}=\widehat{N}_{A}^{X}(a)+(L-a)^{\perp}, \\
& \widehat{N}_{A}^{L}(a)=\widehat{N}_{A}^{X}(a) \cap(L-a), \\
& N_{A}(a)=N_{A}^{L}(a) \oplus(L-a)^{\perp}=N_{A}(a)+(L-a)^{\perp}, \\
& N_{A}^{L}(a)=N_{A}(a) \cap(L-a) .
\end{aligned}
$$

Consequently, the following hold as well:

$$
\begin{aligned}
\widehat{N}_{A}^{X}(a) & =\widehat{N}_{A}^{\operatorname{aff}(A)}(a) \oplus(\operatorname{aff}(A)-a)^{\perp}=\widehat{N}_{A}^{X}(a)+(\operatorname{aff}(A)-a)^{\perp}, \\
\widehat{N}_{A}^{\operatorname{aff}(A)}(a) & =\widehat{N}_{A}^{X}(a) \cap(\operatorname{aff}(A)-a),
\end{aligned}
$$




$$
\begin{aligned}
N_{A}(a) & =N_{A}^{\operatorname{aff}(A)}(a) \oplus(\operatorname{aff}(A)-a)^{\perp}=N_{A}(a)+(\operatorname{aff}(A)-a)^{\perp}, \\
N_{A}^{\operatorname{aff}(A)}(a) & =N_{A}(a) \cap(\operatorname{aff}(A)-a), \\
a \in A \cap B \Rightarrow N_{A}^{\operatorname{aff}(A \cup B)}(a) & =N_{A}(a) \cap \operatorname{span}(A-B) .
\end{aligned}
$$

Proof. (25a): Take $u \in \widehat{N}_{A}^{X}(a)$. Then there exist $\lambda \geq 0, x \in X$, and $a \in P_{A} x$ such that $\lambda(x-a)=u$. Set $b=P_{L} x$. By Lemma 3.3, we have $a \in P_{A} x=\left(P_{A} \circ P_{L}\right) x=P_{A} b$. Using [2, Corollary 3.20(i)], we thus deduce that $\lambda(b-a) \in \widehat{N}_{A}^{L}(a)$ and $\lambda(x-b) \in(L-b)^{\perp}=(L-a)^{\perp}$. Hence $u=\lambda(b-a)+$ $\lambda(x-b) \in \widehat{N}_{A}^{L}(a)+(L-a)^{\perp}=\widehat{N}_{A}^{L}(a) \oplus(L-a)^{\perp}$ by Lemma 3.4(i). We have thus shown that

$$
\widehat{N}_{A}^{X}(a) \subseteq \widehat{N}_{A}^{L}(a) \oplus(L-a)^{\perp} .
$$

On the other hand, Lemma 2.4(iii) implies that $\widehat{N}_{A}^{L}(a) \subseteq \widehat{N}_{A}^{X}(a)$ and thus

$$
\widehat{N}_{A}^{L}(a)+(L-a)^{\perp} \subseteq \widehat{N}_{A}^{X}(a)+(L-a)^{\perp} .
$$

Altogether,

$$
\widehat{N}_{A}^{X}(a) \subseteq \widehat{N}_{A}^{L}(a) \oplus(L-a)^{\perp} \subseteq \widehat{N}_{A}^{X}(a)+(L-a)^{\perp} .
$$

To complete the proof of (25a), it thus suffices to show that $\widehat{N}_{A}^{X}(a)+(L-a)^{\perp} \subseteq \widehat{N}_{A}^{X}(a)$. To this end, let $u \in \widehat{N}_{A}^{X}(a)$ and $v \in(L-a)^{\perp} \subseteq(\operatorname{aff}(A)-a)^{\perp}$. Then there exist $\lambda \geq 0, b \in X$, and $a \in P_{A} b$ such that $u=\lambda(b-a)$. If $\lambda=0$, then $u=0$ and $u+v=v \in(\operatorname{aff}(A)-a)^{\perp} \subseteq(A-a)^{\ominus}=$ $(A-a)^{\ominus} \cap X=(A-a)^{\ominus} \cap \operatorname{cone}(X-a) \subseteq \widehat{N}_{A}^{X}(a)$ by Lemma 2.4(ix)\&(x). Thus, we assume that $\lambda>0$. By Lemma 3.2, we have $a \in P_{A} b=P_{A}\left(b+\lambda^{-1} v\right)$. Hence $b+\lambda^{-1} v-a \in \widehat{N}_{A}^{X}(a)$ and therefore $\lambda\left(b+\lambda^{-1} v-a\right)=\lambda(b-a)+v=u+v \in \widehat{N}_{A}^{X}(a)$, as required.

(25b): By Lemma 2.4(iii)\&(x), $\widehat{N}_{A}^{L}(a) \subseteq \widehat{N}_{A}^{X}(a) \cap(L-a)$. Now let $u \in \widehat{N}_{A}^{X}(a) \cap(L-a)$. By (25a), we have $u=v+w$, where $v \in \widehat{N}_{A}^{L}(a) \subseteq L-a$ and $w \in(L-a)^{\perp}$. On the other hand, $w=u-v \in(L-a)-(L-a)=L-a$. Altogether $w \in(L-a) \cap(L-a)^{\perp}=\{0\}$. Hence $u=v \in \widehat{N}_{A}^{L}(a)$.

(25c): Let $u \in N_{A}(a)$. By definition, there exist sequences $\left(a_{n}\right)_{n \in \mathbb{N}}$ in $A$ and $\left(u_{n}\right)_{n \in \mathbb{N}}$ in $X$ such that $a_{n} \rightarrow a, u_{n} \rightarrow u$, and $(\forall n \in \mathbb{N}) u_{n} \in \widehat{N}_{A}^{X}\left(a_{n}\right)$. By (25a), there exists a sequence $\left(v_{n}, w_{n}\right)_{n \in \mathbb{N}}$ such that $\left(a_{n}, v_{n}\right)_{n \in \mathbb{N}}$ lies in gr $\widehat{N}_{A}^{L},\left(w_{n}\right)_{n \in \mathbb{N}}$ lies in $(L-a)^{\perp}$, and $(\forall n \in \mathbb{N}) u_{n}=v_{n}+w_{n}$ and $v_{n} \perp w_{n}$. Since $\|u\|^{2} \leftarrow\left\|u_{n}\right\|^{2}=\left\|v_{n}\right\|^{2}+\left\|w_{n}\right\|^{2}$, the sequences $\left(v_{n}\right)_{n \in \mathbb{N}}$ and $\left(w_{n}\right)_{n \in \mathbb{N}}$ are bounded. After passing to subsequences and relabeling if necessary, we assume $\left(v_{n}\right)_{n \in \mathbb{N}}$ and $\left(w_{n}\right)_{n \in \mathbb{N}}$ are convergent, with limits $v$ and $w$, respectively. It follows that $v \in N_{A}^{L}(a)$ and $w \in(L-a)^{\perp}$; consequently, $u=v+w \in N_{A}^{L}(a) \oplus(L-a)^{\perp}$ by Lemma 3.4(ii). Thus $N_{A}(a) \subseteq N_{A}^{L}(a) \oplus(L-a)^{\perp}$. On the other hand, by Lemma 2.4(iii), $N_{A}^{L}(a) \oplus(L-a)^{\perp} \subseteq N_{A}(a)+(L-a)^{\perp}$. Altogether,

$$
N_{A}(a) \subseteq N_{A}^{L}(a) \oplus(L-a)^{\perp} \subseteq N_{A}(a)+(L-a)^{\perp} .
$$

It thus suffices to prove that $N_{A}(a)+(L-a)^{\perp} \subseteq N_{A}(a)$. To this end, take $u \in N_{A}(a)$ and $v \in$ $(L-a)^{\perp}$. Then there exist sequences $\left(a_{n}\right)_{n \in \mathbb{N}}$ in $A$ and $\left(u_{n}\right)_{n \in \mathbb{N}}$ in $X$ such that $a_{n} \rightarrow a, u_{n} \rightarrow u$, 
and $(\forall n \in \mathbb{N}) u_{n} \in \widehat{N}_{A}^{X}\left(a_{n}\right)$. For every $n \in \mathbb{N}$, we have $L-a=L-a_{n}$ and hence $u_{n}+v \in$ $\widehat{N}_{A}^{X}\left(a_{n}\right)+\left(L-a_{n}\right)^{\perp}=\widehat{N}_{A}^{X}\left(a_{n}\right)$ by (25a). Passing to the limit, we conclude that $u+v \in N_{A}(a)$.

(25d): First, take $u \in N_{A}^{L}(a)$. On the one hand, by Lemma 2.4(iii), $u \in N_{A}(a)$. On the other hand, by Lemma 3.4(ii), $u \in(L-a)^{\perp \perp}=L-a$. Altogether, we have shown that

$$
N_{A}^{L}(a) \subseteq N_{A}(a) \cap(L-a) .
$$

Conversely, take $u \in N_{A}(a) \cap(L-a) \subseteq N_{A}(a)$. By (25c), there exist $v \in N_{A}^{L}(a)$ and $w \in(L-a)^{\perp}$ such that $u=v+w$ and $v \perp w$. By (31), $v \in L-a$. Hence $w=u-v \in(L-a)-(L-a)=$ $L-a$. Since $w \in(L-a)^{\perp}$, we deduce that $w=0$. This implies $u=v \in N_{A}^{L}(a)$. Therefore, $N_{A}(a) \cap(L-a) \subseteq N_{A}^{L}(a)$.

"Consequently" part: Consider (25) when $L=\operatorname{aff}(A)$ or $L=\operatorname{aff}(A \cup B)$, and recall Lemma 3.1 in the latter case.

An immediate consequence of Theorem 3.5 (or of the definitions) is the following result.

Corollary 3.6 (the $X$-restricted and the Mordukhovich normal cone coincide) Let A be a nonempty subset of $X$, and let $a \in A$. Then

$$
N_{A}^{X}(a)=N_{A}(a)
$$

The next two results provide some useful calculus rules.

Corollary 3.7 (restricted normal cone of a sum) Let $C_{1}$ and $C_{2}$ be nonempty closed convex subsets of $X$, let $a_{1} \in C_{1}$, let $a_{2} \in C_{2}$, and let $L$ be an affine subspace of $X$ containing $C_{1}+C_{2}$. Then

$$
N_{C_{1}+C_{2}}^{L}\left(a_{1}+a_{2}\right)=N_{C_{1}}^{L-a_{2}}\left(a_{1}\right) \cap N_{C_{2}}^{L-a_{1}}\left(a_{2}\right) .
$$

Proof. Set $C=C_{1}+C_{2}$ and $a=a_{1}+a_{2}$. Then (25d) and [17, Exercise 6.44] yield

$$
\begin{aligned}
N_{C}^{L}(a) & =N_{C}(a) \cap(L-a)=N_{C_{1}}\left(a_{1}\right) \cap N_{C_{2}}\left(a_{2}\right) \cap(L-a) \\
& =\left(N_{C_{1}}\left(a_{1}\right) \cap(L-a)\right) \cap\left(N_{C_{2}}\left(a_{2}\right) \cap(L-a)\right) .
\end{aligned}
$$

Note that $L-a$ is a linear subspace of $X$ containing $C_{1}-a_{1}$ and $C_{2}-a_{2}$. Thus, $L-a_{2}=L-a+a_{1}$ is an affine subspace of $X$ containing $C_{1}$, and $L-a_{1}=L-a+a_{2}$ is an affine subspace of $X$ containing $C_{2}$. By $(25 \mathrm{~d})$,

$$
N_{C_{1}}^{L-a_{2}}\left(a_{1}\right)=N_{C_{1}}\left(a_{1}\right) \cap(L-a) \quad \text { and } \quad N_{C_{2}}^{L-a_{1}}\left(a_{2}\right)=N_{C_{2}}\left(a_{2}\right) \cap(L-a) .
$$

The conclusion follows by combining (34) and (35).

Corollary 3.8 (an intersection formula) Let $A$ and $B$ be nonempty closed convex subsets of $X$, and suppose that $a \in A \cap B$. Let $L$ be an affine subspace of $X$ containing $A \cup B$. Then

$$
N_{A}^{L}(a) \cap\left(-N_{B}^{L}(a)\right)=N_{A-B}^{L-a}(0) .
$$


Proof. Using (25d), Proposition 2.7(v), [17, Exercise 6.44], and again (25d), we obtain

$$
\begin{aligned}
N_{A}^{L}(a) \cap\left(-N_{B}^{L}(a)\right) & =N_{A}(a) \cap(L-a) \cap\left(-N_{B}(a)\right) \cap(L-a) \\
& =\left(N_{A}(a) \cap\left(-N_{B}(a)\right)\right) \cap(L-a) \\
& =\left(N_{A}(a) \cap N_{-B}(-a)\right) \cap(L-a) \\
& =N_{A-B}(0) \cap(L-a) \\
& =N_{A-B}^{L-a}(0),
\end{aligned}
$$

as required.

Let us now work towards relating the restricted normal cone to the (relative and classical) interior and to the boundary of a given set.

Proposition 3.9 Let $A$ be a nonempty subset of $X$, let $a \in A$, let $L$ be an affine subspace containing $A$, and suppose that $N_{A}^{L}(a)=\{0\}$. Then $L=\operatorname{aff}(A)$.

Proof. Using $0 \in N_{A}^{\text {aff }(A)}(a) \subseteq N_{A}^{L}(a)=\{0\}$ and applying (25c) and (26c), we have

$$
N_{A}(a)=0+(L-a)^{\perp}=0+(\operatorname{aff}(A)-a)^{\perp} .
$$

So $L-a=\operatorname{aff}(A)-a$, i.e., $L=\operatorname{aff}(A)$.

Theorem 3.10 Let $A$ and $B$ be nonempty subsets of $X$, and let $a \in A$. Then

$$
N_{A}^{B}(a)=\{0\} \quad \Leftrightarrow \quad(\exists \delta>0)(\forall x \in A \cap \operatorname{ball}(a ; \delta)) P_{A}^{-1}(x) \cap B \subseteq\{x\} .
$$

Furthermore, if $A$ is closed and $B$ is an affine subspace of $X$ containing $A$, then the following are equivalent:

(i) $N_{A}^{B}(a)=\{0\}$.

(ii) $(\exists \rho>0) \operatorname{ball}(a ; \rho) \cap B \subseteq A$.

(iii) $B=\operatorname{aff}(A)$ and $a \in \operatorname{ri}(A)$.

Proof. Note that $N_{A}^{B}(a)=\{0\} \Leftrightarrow(\exists \delta>0)(\forall x \in A \cap \operatorname{ball}(a ; \delta)) \widehat{N}_{A}^{B}(x)=\{0\}$. Hence (39) follows from the definition of $\widehat{N}_{A}^{B}(x)$.

Now suppose that $A$ is closed and $B$ is an affine subspace of $X$ containing $A$.

"(i) $\Rightarrow\left(\right.$ ii)": Let $\delta>0$ be as in (39) and set $\rho:=\delta / 2$. Let $b \in B(a ; \rho) \cap B$, and take $x \in P_{A} b$, which is possible since $A$ is closed. Then $\|b-x\|=d_{A}(b) \leq\|b-a\| \leq \rho$ and hence

$$
\|x-a\| \leq\|x-b\|+\|b-a\| \leq \rho+\rho=2 \rho=\delta .
$$

Using (39), we deduce that $b \in P_{A}^{-1}(x) \cap B \subseteq\{x\} \subseteq A$. 
"(ii) $\Rightarrow$ (iii)": It follows that $B=\operatorname{aff}(B) \subseteq \operatorname{aff}(A) \subseteq B$; hence, $B=\operatorname{aff}(A)$. Thus ball $(a ; \rho) \cap$ $\operatorname{aff}(A) \subseteq A$, which means that $a \in \operatorname{ri}(A)$.

“(iii) $\Rightarrow($ i)”: Lemma 2.4(viii).

Corollary 3.11 (interior and boundary characterizations) Let $A$ be a nonempty closed subset of $X$, and let $a \in A$. Then the following hold:

(i) $N_{A}^{\mathrm{aff}(A)}(a)=\{0\} \Leftrightarrow a \in \operatorname{ri}(A)$.

(ii) $N_{A}^{\mathrm{aff}(A)}(a) \neq\{0\} \Leftrightarrow a \in A \backslash \operatorname{ri}(A)$.

(iii) $N_{A}(a)=\{0\} \Leftrightarrow a \in \operatorname{int}(A)$.

(iv) $N_{A}(a) \neq\{0\} \Leftrightarrow a \in A \backslash \operatorname{int}(A)$.

Proof. (i): Apply Theorem 3.10 with $B=\operatorname{aff}(A)$. (ii): Clear from (i). (iii): Apply Theorem 3.10 with $B=X$, and recall Corollary 3.6. (iv): Clear from (iii).

A second look at the proof of (i) $\Rightarrow$ (ii) in Theorem 3.10 reveals that this implication does actually not require the assumption that $B$ be an affine subspace of $X$ containing $A$. The following example illustrates that the converse implication fails even when $B$ is a superset of $\operatorname{aff}(A)$.

Example 3.12 Suppose that $X=\mathbb{R}^{2}$, and set $A:=\mathbb{R} \times\{0\}, a=(0,0)$, and $B=\mathbb{R} \times\{0,2\}$. Then $A=\operatorname{aff}(A) \subseteq B$ and ball $(a ; 1) \cap B \subseteq A$; however, $(\forall x \in A) \widehat{N}_{A}^{B}(x)=\{0\} \times \mathbb{R}_{+}$and therefore $N_{A}^{B}(a)=\{0\} \times \mathbb{R}_{+} \neq\{(0,0)\}$.

\section{Two convex sets}

It is instructive to interpret the previous results for two convex sets:

Theorem 3.13 (two convex sets: restricted normal cones and relative interiors) Let $A$ and $B$ be nonempty convex subsets of $X$. Then the following are equivalent:

(i) ri $A \cap$ ri $B \neq \varnothing$.

(ii) $0 \in \operatorname{ri}(B-A)$.

(iii) $\operatorname{cone}(B-A)=\operatorname{span}(B-A)$.

(iv) $N_{A}(c) \cap\left(-N_{B}(c)\right) \cap \overline{\text { cone }}(B-A)=\{0\}$ for some $c \in A \cap B$.

(v) $N_{A}(c) \cap\left(-N_{B}(c)\right) \cap \overline{\text { cone }}(B-A)=\{0\}$ for every $c \in A \cap B$.

(vi) $N_{A}(c) \cap\left(-N_{B}(c)\right) \cap \operatorname{span}(B-A)=\{0\}$ for some $c \in A \cap B$. 
(vii) $N_{A}(c) \cap\left(-N_{B}(c)\right) \cap \operatorname{span}(B-A)=\{0\}$ for every $c \in A \cap B$.

(viii) $N_{A}^{\mathrm{aff}(A \cup B)}(c) \cap\left(-N_{B}^{\mathrm{aff}(A \cup B)}(c)\right)=\{0\}$ for some $c \in A \cap B$.

(ix) $N_{A}^{\text {aff }(A \cup B)}(c) \cap\left(-N_{B}^{\text {aff }(A \cup B)}(c)\right)=\{0\}$ for every $c \in A \cap B$.

(x) $N_{A-B}^{\mathrm{span}(B-A)}(0)=\{0\}$.

Proof. By [16, Corollary 6.6.2], (ii) $\Leftrightarrow$ ri $A \cap$ ri $B \neq \varnothing \Leftrightarrow 0 \in$ ri $A-$ ri $B \Leftrightarrow$ (ii).

Applying Proposition 1.7 to $B-A$, and [1, Proposition 3.1.3] to $\overline{\text { cone }}(B-A)$, we obtain

$$
\text { (ii) } \begin{aligned}
& \Leftrightarrow(\text { iii }) \Leftrightarrow \overline{\text { cone }}(B-A)=\operatorname{span}(B-A) \\
& \Leftrightarrow \overline{\text { cone }}(B-A) \cap(\overline{\text { cone }}(B-A))^{\oplus}=\{0\} .
\end{aligned}
$$

Let $c \in A \cap B$. Then Corollary 3.8 (with $L=X)$ yields $N_{A}(c) \cap\left(-N_{B}(c)\right)=N_{A-B}(0)=(A-$ $B)^{\ominus}=(B-A)^{\oplus}=(\overline{\text { cone }}(B-A))^{\oplus}$. Hence

$$
(\forall c \in C) \quad N_{A}(c) \cap\left(-N_{B}(c)\right) \cap \overline{\text { cone }}(B-A)=(\overline{\text { cone }}(B-A))^{\oplus} \cap \overline{\text { cone }}(B-A)
$$

and

$$
(\forall c \in C) \quad N_{A}(c) \cap\left(-N_{B}(c)\right) \cap \operatorname{span}(B-A)=(\overline{\text { cone }}(B-A))^{\oplus} \cap \operatorname{span}(B-A) .
$$

Combining (41), (42), and (43), we see that (ii)-(vii) are equivalent.

Next, Lemma 3.1 and Corollary 3.8 yield the equivalence of (viii)-(x).

Finally, (x) $\Leftrightarrow($ ii) by Corollary 3.11(i).

Corollary 3.14 (two convex sets: normal cones and interiors) Let $A$ and $B$ be nonempty convex subsets of $X$. Then the following are equivalent:

(i) $0 \in \operatorname{int}(B-A)$.

(ii) $\operatorname{cone}(B-A)=X$.

(iii) $N_{A}(c) \cap\left(-N_{B}(c)\right)=\{0\}$ for some $c \in A \cap B$.

(iv) $N_{A}(c) \cap\left(-N_{B}(c)\right)=\{0\}$ for every $c \in A \cap B$.

(v) $N_{A-B}(0)=\{0\}$.

Proof. We start by notating that if $C$ is a convex subset of $X$, then $0 \in \operatorname{int} C \Leftrightarrow 0 \in \operatorname{ri} C$ and span $C=X$. Consequently,

$$
\text { (i) } \Leftrightarrow \quad \Leftrightarrow \in \operatorname{ri}(B-A) \text { and } \operatorname{span}(B-A)=X \text {. }
$$

Assume that (i) holds. Then (44) and Theorem 3.13 imply that cone $(B-A)=\overline{\text { cone }}(B-A)=$ $\operatorname{span}(B-A)=X$. Hence (ii) holds, and from Theorem 3.13 we obtain that (ii) $\Rightarrow($ iii) $\Leftrightarrow(\mathrm{iv}) \Leftrightarrow(\mathrm{v})$. Finally, Corollary 3.11(iii) yields the implication $(v) \Rightarrow(\mathrm{i})$. 


\section{Further examples}

In this section, we provide further examples that illustrate particularities of restricted normal cones.

As announced in Remark 2.8, when $a \in A_{2} \varsubsetneqq A_{1}$, it is possible that the nonconvex restricted normal cones satisfy $N_{A_{1}}^{B}(a) \nsubseteq N_{A_{2}}^{B}(a)$ even when $A_{1}$ and $A_{2}$ are both convex. This lack of inclusion is also known for the Mordukhovich normal cone (see [15, page 5], where however one of the sets is not convex). Furthermore, the following example also shows that the restricted normal cone cannot be derived from the Mordukhovich normal cone by the simple relativization procedure of intersecting with naturally associated cones and subspaces.

Example 4.1 (lack of convexity, inclusion, and relativization) Suppose that $X=\mathbb{R}^{2}$, and define two nonempty closed convex sets by $A:=A_{1}:=\operatorname{epi}(|\cdot|)$ and $A_{2}:=\operatorname{epi}(2|\cdot|)$. Then $a:=(0,0) \in$ $A_{2} \varsubsetneqq A_{1}$. Furthermore, set $B:=\mathbb{R} \times\{0\}$. Then

$$
\begin{aligned}
& \left(\forall x=\left(x_{1}, x_{2}\right) \in A_{1}\right) \quad \widehat{N}_{A_{1}}^{B}(x)= \begin{cases}\mathbb{R}_{+}(1,-1), & \text { if } x_{2}=x_{1}>0 ; \\
\mathbb{R}_{+}(-1,-1), & \text { if } x_{2}=-x_{1}>0 ; \\
\{(0,0)\}, & \text { otherwise }\end{cases} \\
& \left(\forall x=\left(x_{1}, x_{2}\right) \in A_{2}\right) \quad \widehat{N}_{A_{2}}^{B}(x)= \begin{cases}\mathbb{R}_{+}(2,-1), & \text { if } x_{2}=2 x_{1}>0 ; \\
\mathbb{R}_{+}(-2,-1), & \text { if } x_{2}=-2 x_{1}>0 ; \\
\{(0,0)\}, & \text { otherwise. }\end{cases}
\end{aligned}
$$

Consequently,

$$
\begin{aligned}
& N_{A_{1}}^{B}(a)=\text { cone }\{(1,-1),(-1,-1)\}, \\
& N_{A_{2}}^{B}(a)=\text { cone }\{(2,-1),(-2,-1)\} .
\end{aligned}
$$

Note that $N_{A_{1}}^{B}(a) \nsubseteq N_{A_{2}}^{B}(a)$ and $N_{A_{2}}^{B}(a) \nsubseteq N_{A_{1}}^{B}(a)$; in fact, $N_{A_{1}}^{B}(a) \cap N_{A_{2}}^{B}(a)=\{(0,0)\}$. Furthermore, neither $N_{A_{1}}^{B}(a)$ nor $N_{A_{2}}^{B}(a)$ is convex even though $A_{1}, A_{2}$, and $B$ are. Finally, observe that cone $(B-a)=\operatorname{span}(B-a)=B$, that cone $(B-A)=\mathbb{R} \times \mathbb{R}_{-}$, that $\operatorname{span}(B-A)=X$, and that $N_{A}(a)=$ cone $[(1,-1),(-1,-1)] \neq N_{A}^{B}(a)$. Consequently, cone $(B-a) \cap N_{A}(a)=$ $\operatorname{span}(B-a) \cap N_{A}(a)=\{(0,0)\}, \operatorname{cone}(B-A) \cap N_{A}(a)=N_{A}(a)=\operatorname{span}(B-A) \cap N_{A}(a)$. Therefore, $N_{A}^{B}(a)$ cannot be obtained by intersecting the Mordukhovich normal cone with one of the sets cone $(B-a), \operatorname{span}(B-a), \operatorname{cone}(B-A)$, and $\operatorname{span}(B-A)$.

We shall present some further examples. The proof of the following result is straightforward and hence omitted.

Proposition 4.2 Let $K$ be a closed cone in $X$, and let $B$ be a nonempty cone of $X$. Then

$$
N_{K}^{B}(0)=\overline{\bigcup_{x \in K} \widehat{N}_{K}^{B}(x)}=\overline{\bigcup_{x \in \text { bdry } K} \widehat{N}_{K}^{B}(x)}=\overline{\bigcup_{x \in K} N_{K}^{B}(x)}=\overline{\bigcup_{x \in \text { bdry } K} N_{K}^{B}(x)} .
$$


Example 4.3 Let $K$ be a closed convex cone in $X$, suppose that $u_{0} \in \operatorname{int}(K)$ and that $K \subseteq\left\{u_{0}\right\}^{\oplus}$, and set $B:=\left\{u_{0}\right\}^{\perp}$. Then:

(i) $(\forall x \in K \cap B) \widehat{N}_{K}^{B}(x)=\{0\}$.

(ii) $(\forall x \in K \backslash B) \widehat{N}_{K}^{B}(x)=N_{K}^{B}(x)=N_{K}(x)=K^{\ominus} \cap\{x\}^{\perp}$.

(iii) $N_{K}^{B}(0)=\overline{\bigcup_{x \in K} \widehat{N}_{K}^{B}(x)}=\overline{\bigcup_{x \in K \backslash B}\left(K^{\ominus} \cap\{x\}^{\perp}\right)}=\overline{K^{\ominus} \cap \bigcup_{x \in K \backslash B}\{x\}^{\perp}}$.

If one of these unions is closed, then all closures may be omitted.

Proof. (i): Let $x \in K \cap B$. It suffices to show that $B \cap P_{K}^{-1}(x)=\{x\}$. To this end, take $y \in B \cap P_{K}^{-1}(x)$. By definition of $B$, we have $\left\langle u_{0}, x\right\rangle=0$ and $\left\langle u_{0}, y\right\rangle=0$. Hence

$$
\left\langle u_{0}, y-x\right\rangle=0 \text {. }
$$

Furthermore, $x=P_{K} y$ and hence, using e.g. [2, Proposition 6.27], we have $y-x \in K^{\ominus}$. Since $u_{0} \in \operatorname{int} K$, there exists $\delta>0$ such that ball $\left(u_{0} ; \delta\right) \subseteq K$. Thus $y-x \in\left(\text { ball }\left(u_{0} ; \delta\right)\right)^{\ominus}$. In view of $(48)$, $\delta\|y-x\| \leq 0$. Therefore, $y=x$.

(ii): Let $x \in K \backslash B$. Using Lemma 2.4(iii)\&(iv), Corollary 3.6, Lemma 2.4(vii), and [2, Example 6.39], we have

$$
\widehat{N}_{K}^{B}(x) \subseteq \widehat{N}_{K}^{X}(x) \subseteq N_{K}^{X}(x)=N_{K}(x)=N_{K}^{\text {conv }}(x)=K^{\ominus} \cap\{x\}^{\perp} .
$$

Since $x \in K \subseteq\left\{u_{0}\right\}^{\oplus}$ and $x \notin B$, we have $\left\langle u_{0}, x\right\rangle>0$. Now take $u \in\left(K^{\ominus} \cap\{x\}^{\perp}\right) \backslash\{0\}$. Since $u \in K^{\ominus}$ and $u_{0} \in \operatorname{int}(K)$, we have $\left\langle u, u_{0}\right\rangle<0$. Now set

$$
b:=x-\frac{\left\langle u_{0}, x\right\rangle}{\left\langle u_{0}, u\right\rangle} u
$$

Then $b \in B$ and $b-x=-\left\langle u_{0}, x\right\rangle\left\langle u_{0}, u\right\rangle^{-1} u \in \mathbb{R}_{++} u \subseteq K^{\ominus} \cap\{x\}^{\perp}=N_{K}^{\text {conv }}(x)$. By [2, Proposition 6.46], $x=P_{K} b$. Hence $b-x \in \widehat{N}_{K}^{B}(x)$ and thus $u \in \widehat{N}_{K}^{B}(x)$. Therefore, $K^{\ominus} \cap\{x\}^{\perp} \subseteq \widehat{N}_{K}^{B}(x)$. In view of (49), and since $\widehat{N}_{K}^{B}(x) \subseteq N_{K}^{B}(x) \subseteq N_{K}(x)$ by Lemma 2.4(iii)\&(iv), we have established (ii).

(iii): Combine (i), (ii), and Proposition 4.2.

Example 4.4 (ice cream cone) Suppose that $X=\mathbb{R}^{m}=\mathbb{R}^{m-1} \times \mathbb{R}$, where $m \in\{2,3,4, \ldots\}$, and let $\beta>0$. Define the corresponding closed convex ice cream cone by

$$
K:=\left\{x \in \mathbb{R}^{m} \mid \beta \sqrt{x_{1}^{2}+\cdots+x_{m-1}^{2}} \leq x_{m}\right\},
$$

and set $B:=\mathbb{R}^{m-1} \times\{0\}$. Then the following hold:

(i) $\widehat{N}_{K}^{B}(0,0)=\{(0,0)\}$. 
(ii) $N_{K}(0,0)=\left\{y \in \mathbb{R}^{m} \mid \beta^{-1} \sqrt{y_{1}^{2}+\cdots+y_{m-1}^{2}} \leq-y_{m}\right\}=\bigcup_{\substack{z \in \mathbb{R}^{m-1}\|z\| \leq 1 \\ \mathbb{R}_{+}}}(\beta z,-1)$.

(iii) $\left(\forall z \in \mathbb{R}^{m-1} \backslash\{0\}\right) \widehat{N}_{K}^{B}(z, \beta\|z\|)=N_{K}^{B}(z, \beta\|z\|)=N_{K}(z, \beta\|z\|)=\mathbb{R}_{+}(\beta z,-\|z\|)$.

(iv) $N_{K}^{B}(0,0)=\bigcup_{\substack{z \in \mathbb{R}^{m-1} \\\|z\|=1}} \mathbb{R}_{+}(\beta z,-1)$, which is a closed cone that is not convex.

Proof. Clearly, $K$ is closed and convex. Note that $K$ is the lower level set of height 0 of the continuous convex function

$$
f: \mathbb{R}^{m}=\mathbb{R}^{m-1} \times \mathbb{R} \rightarrow \mathbb{R}: x=\left(z, x_{m}\right) \mapsto \beta\|z\|-x_{m} ;
$$

hence , by [20, Exercise 2.5(b) and its solution on page 205],

$$
\operatorname{int}(K)=\left\{x=\left(z, x_{m}\right) \in \mathbb{R}^{m-1} \times \mathbb{R} \mid \beta\|z\|<x_{m}\right\} .
$$

Lemma 2.4(iii)\&(iv), Corollary 3.6, and Corollary 3.11(iii) imply that

$$
(\forall x \in \operatorname{int}(K)) \quad \widehat{N}_{K}^{B}(x) \subseteq \widehat{N}_{K}^{X}(x) \subseteq N_{K}^{X}(x)=N_{K}(x)=\{0\} .
$$

Write $x=\left(z, x_{m}\right) \in \mathbb{R}^{m-1} \times \mathbb{R}=X$, and assume that $x \in K$. We thus assume that $x \in \operatorname{bdry}(K)$, i.e., $\beta\|z\|=x_{m}$ by (53), i.e., $x=(z, \beta\|z\|)$. Combining [2, Proposition 16.8] with [20, Corollary 2.9.5] (or [2, Lemma 26.17]) applied to $f$, we obtain

$$
N_{K}(z, \beta\|z\|)=\text { cone }(\beta \partial\|\cdot\|(z) \times\{-1\}),
$$

where $\partial\|\cdot\|$ denotes the subdifferential operator from convex analysis applied to the Euclidean norm in $\mathbb{R}^{m-1}$. In view of [2, Example 16.25] we thus have

$$
N_{K}(z, \beta\|z\|)= \begin{cases}\text { cone }\left(\beta\|z\|^{-1} z \times\{-1\}\right), & \text { if } z \neq 0 \\ \text { cone }(\operatorname{ball}(0 ; \beta) \times\{-1\}), & \text { if } z=0 .\end{cases}
$$

The case $z=0$ in (56) readily leads to (ii).

Now set $u_{0}:=(0,1) \in \mathbb{R}^{m-1} \times \mathbb{R}$. Then $\left\{u_{0}\right\}^{\perp}=B$ and $\left\{u_{0}\right\}^{\oplus}=\mathbb{R}^{m-1} \times \mathbb{R}_{+} \supseteq K$. Note that $(0,0) \in K \cap B$ and thus $\widehat{N}_{K}^{B}(0,0)=\{(0,0)\}$ by Example 4.3(i). We have thus established (i).

Now assume that $z \neq 0$. Then $N_{K}(z, \beta\|z\|)=\mathbb{R}_{+}(\beta z,-\|z\|)$. Note that $\beta z \neq 0$ and so $(z, \beta\|z\|) \notin B$. The formulas announced in (iii) therefore follow from Example 4.3(ii).

Next, combining (53), (54), and Example 4.3(iii) as well as utilizing the compactness of the unit sphere in $\mathbb{R}^{m-1}$, we see that

$$
N_{K}^{B}(0,0)=\overline{\bigcup_{z \in \mathbb{R}^{m-1} \backslash\{0\}} \mathbb{R}_{+}(\beta z,-\|z\|)}=\overline{\bigcup_{\substack{z \in \mathbb{R}^{m-1} \\\|z\|=1}} \mathbb{R}_{+}(\beta z,-1)}=\bigcup_{\substack{z \in \mathbb{R}^{m-1} \\\|z\|=1}} \mathbb{R}_{+}(\beta z,-1) .
$$

This establishes (iv).

Remark 4.5 Consider Example 4.4. Note that $N_{K}^{B}(0,0)$ is actually the boundary of $N_{K}(0,0)$. Furthermore, since $N_{K}(0,0)=N_{K}^{\text {conv }}(0,0)$ by Lemma $2.4\left(\right.$ vii), the formulas in (ii) also describe $K^{\ominus}$, which is therefore an ice cream cone as well. 


\section{Cones containing restricted normal cones}

In this section, we provide various examples illustrating that the restricted (proximal) normal cone does not naturally arise by considering various natural cones containing it.

Let $A$ and $B$ be nonempty subsets of $X$, and let $a \in A$. We saw in Lemma 2.4(ii) that

$$
\widehat{N}_{A}^{B}(a)=\text { cone }\left((B-a) \cap\left(P_{A}^{-1} a-a\right)\right) \subseteq \operatorname{cone}(B-a) \cap N_{A}^{\text {prox }}(a) .
$$

This raises the question whether or not the inclusion in (58) is strict. It turns out and as we shall now illustrate, both conceivable alternatives (equality and strict inclusion) do occur. Therefore, $\widehat{N}_{A}^{B}(a)$ is a new construction.

We start with a condition sufficient for equality in (58),

Proposition 5.1 Let $A$ and $B$ be nonempty subsets of $X$. Let $A$ be closed and $a \in A$. Assume that one of the following holds:

(i) $P_{A}^{-1}(a)-a$ is a cone.

(ii) $A$ is convex.

Then $\widehat{N}_{A}^{B}(a)=\operatorname{cone}(B-a) \cap N_{A}^{\text {prox }}(a)$.

Proof. (i): Lemma 1.5(ii). (ii): Combine (i) with Lemma 1.6.

The next examples illustrates that equality in (58) can occur even though $P_{A}^{-1}(a)-a$ is not a cone. Consequently, the assumption that $P_{A}^{-1}(a)-a$ be a cone in Proposition 5.1 is sufficient-but not necessary-for equality in (58).

Example 5.2 Suppose that $X=\mathbb{R}^{2}$, and let $A:=X \backslash \mathbb{R}_{++}^{2}, B:=\mathbb{R}_{+}(1,1)$, and $a:=(0,1)$. Then one verifies that

$$
\begin{aligned}
P_{A}^{-1}(a)-a & =[0,1] \times\{0\}, \\
N_{A}^{\text {prox }}(a) & =\text { cone }\left(P_{A}^{-1} a-a\right)=\mathbb{R}_{+} \times\{0\}, \\
\operatorname{cone}(B-a) & =\left\{\left(t_{1}, t_{2}\right) \in \mathbb{R}^{2} \mid t_{1} \geq 0, t_{2}<t_{1}\right\} \cup\{(0,0)\}, \\
\widehat{N}_{A}^{B}(a) & =\mathbb{R}_{+} \times\{0\} .
\end{aligned}
$$

Hence $\widehat{N}_{A}^{B}(a)=\mathbb{R}_{+} \times\{0\}=\operatorname{cone}(B-a) \cap N_{A}^{\text {prox }}(a)$.

We now provide an example where the inclusion in (58) is strict. 
Example 5.3 Suppose that $X=\mathbb{R}^{2}$, let $A:=\operatorname{cone}\{(1,0),(0,1)\}=\operatorname{bdry} \mathbb{R}_{+}^{2}, B:=\mathbb{R}_{+}(2,1)$, and $a:=(0,1) \in A$. Then one verifies that

$$
\begin{aligned}
P_{A}^{-1}(a)-a & =]-\infty, 1] \times\{0\}, \\
N_{A}^{\text {prox }}(a) & =\operatorname{cone}\left(P_{A}^{-1} a-a\right)=\mathbb{R} \times\{0\}, \\
\operatorname{cone}(B-a) & =\left\{\left(x_{1}, x_{2}\right) \in \mathbb{R}^{2} \mid x_{1} \geq 0,2 x_{2}<x_{1}\right\} \cup\{(0,0)\}, \\
\widehat{N}_{A}^{B}(a) & =\{(0,0)\} .
\end{aligned}
$$

Hence $\widehat{N}_{A}^{B}(a)=\{(0,0)\} \varsubsetneqq \mathbb{R}_{+} \times\{0\}=\operatorname{cone}(B-a) \cap N_{A}^{\text {prox }}(a)$, and therefore the inclusion in (58) is strict. In accordance with Proposition 5.1, neither is $P_{A}^{-1}(a)-a$ a cone nor is $A$ convex.

Let us now turn to the restricted normal cone $N_{A}^{B}(a)$. Taking the outer limit in (58) and recalling (13), we obtain

$$
\begin{aligned}
N_{A}^{B}(a) & =\varlimsup_{\substack{x \rightarrow a \\
x \in A}} \widehat{N}_{A}^{B}(x) \\
& \subseteq \varlimsup_{\substack{x \rightarrow a \\
x \in A}}\left(\operatorname{cone}(B-x) \cap N_{A}^{\text {prox }}(x)\right) \\
& \subseteq\left(\varlimsup_{\substack{x \rightarrow a \\
x \in A}} \operatorname{cone}(B-x)\right) \cap N_{A}(a) .
\end{aligned}
$$

The inclusions in (61) are optimal in the sense that all possible combinations (strict inclusion and equality) can occur:

- For results and examples illustrating equality in (61b) and equality in (61c), see Proposition 5.5 and Example 5.6 below.

- For an example illustrating equality in (61b) and strict inequality in (61c), see Example 5.7 below.

- For an example illustrating strict inequality in (61b) and equality in (61c), see Example 5.10 below.

- For examples illustrating strict inequality in (61b) and strict inequality in (61c), see Example 5.8 and Example 5.9 below.

The remainder of this section is devoted to providing these examples.

Proposition 5.4 Let $A$ and $B$ be nonempty subsets of X. Let $A$ be closed $a \in A$. Assume that one of the following holds:

(i) $P_{A}^{-1}(x)-x$ is a cone for every $x \in A$ sufficiently close to $a$.

(ii) $A$ is convex. 
Then (61b) holds with equality, i.e., $N_{A}^{B}(a)=\varlimsup_{\substack{x \rightarrow a \\ x \in A}}\left(\operatorname{cone}(B-x) \cap N_{A}^{\text {prox }}(x)\right)$

Proof. Indeed, if $x \in A$ is sufficiently close to $a$, then Proposition 5.1 implies that $\widehat{N}_{A}^{B}(x)=\operatorname{cone}(B-$ $x) \cap N_{A}^{\text {prox }}(x)$. Now take the outer limit as $x \rightarrow a$ in $A$.

Proposition 5.5 Let $A$ be a nonempty closed convex subset of $X$, let $B$ be a nonempty subset of $X$, and let $a \in A$. Assume that $x \mapsto \operatorname{cone}(B-x)$ is outer semicontinuous at a relative to $A$, i.e.,

$$
\varlimsup_{\substack{x \rightarrow a \\ x \in A}} \operatorname{cone}(B-x)=\operatorname{cone}(B-a),
$$

Then (61) holds with equalities, i.e.,

$$
N_{A}^{B}(a)=\varlimsup_{\substack{x \rightarrow a \\ x \in A}}\left(\operatorname{cone}(B-x) \cap N_{A}^{\mathrm{prox}}(x)\right)=\left(\varlimsup_{\substack{x \rightarrow a \\ x \in A}} \operatorname{cone}(B-x)\right) \cap N_{A}(a) .
$$

Proof. The convexity of $A$ and Lemma 2.4(vii) yield

$$
\operatorname{cone}(B-a) \cap N_{A}(a)=\operatorname{cone}(B-a) \cap N_{A}^{\text {prox }}(a) .
$$

On the other hand, Proposition 5.1(ii) and Lemma 2.4(iv) imply

$$
\operatorname{cone}(B-a) \cap N_{A}^{\text {prox }}(a)=\widehat{N}_{A}^{B}(a) \subseteq N_{A}^{B}(a) .
$$

Altogether, cone $(B-a) \cap N_{A}(a) \subseteq N_{A}^{B}(a)$. In view of (62),

$$
\left(\varlimsup_{\substack{x \rightarrow a \\ x \in A}} \operatorname{cone}(B-x)\right) \cap N_{A}(a) \subseteq N_{A}^{B}(a) .
$$

Recalling (61), we therefore obtain (63).

Example 5.6 Let $A$ be a linear subspace of $X$, set $B:=A$, and $a:=(0,0)$. Then $N_{A}^{B}(a)=\{0\}$ by $(25 \mathrm{~d}), N_{A}(a)=A^{\perp}$, and cone $(B-x)=A$, for every $x \in A$. Hence $\left(\varlimsup_{\substack{x \rightarrow a \\ x \in A}} \operatorname{cone}(B-x)\right) \cap N_{A}(a)=$ $\{0\}$ and (61) holds with equalities.

In Proposition 5.5, the convexity and the outer semicontinuity assumptions are both essential in the sense that absence of either assumption may make the inclusion (61c) strict; we shall illustrate this in the next three examples.

Example 5.7 Suppose that $X=\mathbb{R}^{2}$, and let $A:=\operatorname{epi}(|\cdot|), B:=\mathbb{R} \times\{0\}$, and $a:=(0,0)$. If $x=$ $\left(x_{1}, x_{2}\right) \in A \backslash\{a\}$, then $x_{2}>0, B-x=\mathbb{R} \times\left\{-x_{2}\right\}$, and so cone $(B-x)=\mathbb{R} \times \mathbb{R}_{--} \cup\{(0,0)\}$. Hence

$$
\varlimsup_{\substack{x \rightarrow a \\ x \in A}} \operatorname{cone}(B-x)=\mathbb{R} \times \mathbb{R}_{-} \neq \mathbb{R} \times\{0\}=\operatorname{cone}(B-a)
$$


i.e., (62) fails. Since $A$ is closed and convex, Lemma $2.4\left(\right.$ vii) implies that $N_{A}(a)=N_{A}^{\text {conv }}(a)=-A$. Thus

$$
\left(\varlimsup_{\substack{x \rightarrow a \\ x \in A}} \operatorname{cone}(B-x)\right) \cap N_{A}(a)=-A .
$$

Proposition 5.4(ii) yields equality in (61b), i.e.,

$$
N_{A}^{B}(a)=\varlimsup_{\substack{x \rightarrow a \\ x \in A}}\left(\operatorname{cone}(B-x) \cap N_{A}^{\text {prox }}(x)\right) .
$$

We observed already in Example 4.1 that

$$
N_{A}^{B}(a)=\text { cone }\{(1,-1),(-1,-1)\} .
$$

Therefore we have

$$
N_{A}^{B}(a)=\varlimsup_{\substack{x \rightarrow a \\ x \in A}}\left(\operatorname{cone}(B-x) \cap N_{A}^{\operatorname{prox}}(x)\right) \varsubsetneqq\left(\varlimsup_{\substack{x \rightarrow a \\ x \in A}} \operatorname{cone}(B-x)\right) \cap N_{A}(a),
$$

i.e., the inclusion (61c) is strict.

Example 5.8 Suppose that $X=\mathbb{R}^{2}$, and let $A:=\operatorname{cone}\{(1,0),(0,1)\}=\operatorname{bdry} \mathbb{R}_{+}^{2}, B:=\mathbb{R} \times\{1\} \cup$ $\{(1,0),(-1,0)\}$, and $a:=(0,0)$. Clearly, $A$ is not convex. If $x=\left(x_{1}, x_{2}\right) \in A$ is sufficiently close to $a$, we have

$$
\operatorname{cone}(B-x)= \begin{cases}\mathbb{R} \times \mathbb{R}_{+}, & \text {if } x_{1} \geq 0 \\ \mathbb{R} \times \mathbb{R}_{++} \cup \operatorname{cone}\left\{\left(1,-x_{2}\right),\left(-1,-x_{2}\right)\right\}, & \text { if } x_{2}>0\end{cases}
$$

This yields

$$
\varlimsup_{\substack{x \rightarrow a \\ x \in A}} \operatorname{cone}(B-x)=\mathbb{R} \times \mathbb{R}_{+}=\operatorname{cone}(B-a),
$$

i.e., (62) holds. Next, if $x=\left(x_{1}, x_{2}\right) \in A$, then

$$
P_{A}^{-1}(x)= \begin{cases}\left.\left.\left\{x_{1}\right\} \times\right]-\infty, x_{1}\right], & \text { if } x_{1}>0 \text { and } x_{2}=0 \\ ]-\infty, x_{2}\right] \times\left\{x_{2}\right\}, & \text { if } x_{1}=0 \text { and } x_{2}>0 \\ \mathbb{R}_{-,}^{2}, & \text { if } x_{1}=x_{2}=0\end{cases}
$$

and so

$$
N_{A}^{\text {prox }}(x)=\text { cone }\left(P_{A}^{-1}(x)-x\right)= \begin{cases}\{0\} \times \mathbb{R}, & \text { if } x_{1}>0 \text { and } x_{2}=0 \\ \mathbb{R} \times\{0\}, & \text { if } x_{1}=0 \text { and } x_{2}>0 \\ \mathbb{R}_{-}^{2}, & \text { if } x_{1}=x_{2}=0\end{cases}
$$

It follows that

$$
N_{A}(a)=\varlimsup_{\substack{x \rightarrow a \\ x \in A}} N_{A}^{\text {prox }}(x)=\mathbb{R}_{-}^{2} \cup(\{0\} \times \mathbb{R}) \cup(\mathbb{R} \times\{0\})
$$


If $x \in A$ is sufficiently close $a$, then

$$
\widehat{N}_{A}^{B}(x)= \begin{cases}\{(0,0)\}, & \text { if } x \neq a \\ \mathbb{R}_{-} \times\{0\}, & \text { if } x=a .\end{cases}
$$

It follows that

$$
N_{A}^{B}(a)=\mathbb{R}_{-} \times\{0\} .
$$

Combining (72) and (75), we obtain for every $x=\left(x_{1}, x_{2}\right) \in A$ sufficiently close to $a$ that

$$
\text { cone }(B-x) \cap N_{A}^{\text {prox }}(x)= \begin{cases}\{0\} \times \mathbb{R}_{+}, & \text {if } x_{1}>0 \text { and } x_{2}=0 ; \\ \{(0,0)\}, & \text { if } x_{1}=0 \text { and } x_{2}>0 ; \\ \mathbb{R}_{-} \times\{0\}, & \text { if } x_{1}=x_{2}=0\end{cases}
$$

Thus

$$
\varlimsup_{\substack{x \rightarrow a \\ x \in A}}\left(\operatorname{cone}(B-x) \cap N_{A}^{\text {prox }}(x)\right)=\left(\{0\} \times \mathbb{R}_{+}\right) \cup\left(\mathbb{R}_{-} \times\{0\}\right) .
$$

Using (78), (80), (73), and (76), we conclude that

$$
\begin{aligned}
N_{A}^{B}(a) & =\mathbb{R}_{-} \times\{0\} \\
& \varsubsetneqq\left(\{0\} \times \mathbb{R}_{+}\right) \cup\left(\mathbb{R}_{-} \times\{0\}\right)=\varlimsup_{\substack{a^{\prime} \rightarrow a \\
a^{\prime} \in A}}\left(\operatorname{cone}(B-x) \cap N_{A}^{\text {prox }}(x)\right) \\
& \varsubsetneqq\left(\{0\} \times \mathbb{R}_{+}\right) \cup(\mathbb{R} \times\{0\})=\left(\varlimsup_{\substack{x \rightarrow a \\
x \in A}} \operatorname{cone}(B-x)\right) \cap N_{A}(a) .
\end{aligned}
$$

Therefore, both inclusions in (61) are strict; however, $A$ is not convex while (62) does hold.

Example 5.9 Suppose that $X=\mathbb{R}^{2}$, let $A:=\operatorname{cone}\{(1,0),(0,1)\}=\operatorname{bdry} \mathbb{R}_{+}^{2}, B:=\mathbb{R}_{+}(2,1)$ and $a:=(0,0)$. Let $x=\left(x_{1}, x_{2}\right) \in A$. Then (see Example 5.8)

$$
\begin{gathered}
P_{A}^{-1}(x)-x= \begin{cases}\left.\{0\} \times]-\infty, x_{1}\right], & \text { if } x_{1}>0 \text { and } x_{2}=0 ; \\
]-\infty, x_{2}\right] \times\{0\}, & \text { if } x_{1}=0 \text { and } x_{2}>0 \\
\mathbb{R}_{-}^{2}, & \text { if } x_{1}=x_{2}=0\end{cases} \\
N_{A}^{\text {prox }}(x)= \begin{cases}\{0\} \times \mathbb{R}, & \text { if } x_{1}>0 \text { and } x_{2}=0 ; \\
\mathbb{R} \times\{0\}, & \text { if } x_{1}=0 \text { and } x_{2}>0 ; \\
\mathbb{R}_{-}^{2}, & \text { if } x_{1}=x_{2}=0\end{cases}
\end{gathered}
$$

and

$$
N_{A}(a)=\varlimsup_{\substack{x \rightarrow a \\ x \in A}} N_{A}^{\text {prox }}(x)=\mathbb{R}_{-}^{2} \cup(\{0\} \times \mathbb{R}) \cup(\mathbb{R} \times\{0\}) .
$$


Thus

$$
\widehat{N}_{A}^{B}(x)=\text { cone }\left(\left(P_{A}^{-1}(x)-x\right) \cap(B-x)\right)= \begin{cases}\{0\} \times \mathbb{R}_{+}, & \text {if } x_{1}>0 \text { and } x_{2}=0 \\ \{(0,0)\}, & \text { if } x_{1}=0 \text { and } x_{2} \geq 0\end{cases}
$$

Hence

$$
N_{A}^{B}(a)=\varlimsup_{\substack{x \rightarrow a \\ x \in A}} \widehat{N}_{A}^{B}(x)=\{0\} \times \mathbb{R}_{+} .
$$

On the other hand,

$$
\operatorname{cone}(B-x)= \begin{cases}\left\{\left(y_{1}, y_{2}\right) \mid y_{2} \geq 0, y_{1}<2 y_{2}\right\} \cup\{(0,0)\}, & \text { if } x_{1}>0 \text { and } x_{2}=0 \\ \left\{\left(y_{1}, y_{2}\right) \mid y_{1} \geq 0,2 y_{2}<y_{1}\right\} \cup\{(0,0)\}, & \text { if } x_{1}=0 \text { and } x_{2}>0 \\ B, & \text { if } x_{1}=x_{2}=0\end{cases}
$$

Combining (83) and (87), we deduce that

$$
\text { cone }(B-x) \cap N_{A}^{\text {prox }}(x)= \begin{cases}\{0\} \times \mathbb{R}_{+}, & \text {if } x_{1}>0 \text { and } x_{2}=0 ; \\ \mathbb{R}_{+} \times\{0\}, & \text { if } x_{1}=0 \text { and } x_{2}>0 ; \\ \{(0,0)\}, & \text { if } x_{1}=x_{2}=0 .\end{cases}
$$

Using (87) and (88), we compute

$$
\varlimsup_{\substack{x \rightarrow a \\ x \in A}} \operatorname{cone}(B-x)=\left\{\left(y_{1}, y_{2}\right) \mid y_{1} \geq 0 \text { or } y_{2} \geq 0\right\}=X \backslash \mathbb{R}_{--}^{2} \neq B=\operatorname{cone}(B-a)
$$

and

$$
\varlimsup_{\substack{x \rightarrow a \\ x \in A}}\left(\operatorname{cone}(B-x) \cap N_{A}^{\text {prox }}(x)\right)=\left(\{0\} \times \mathbb{R}_{+}\right) \cup\left(\mathbb{R}_{+} \times\{0\}\right)=\text { cone }\{(0,1),(1,0)\} .
$$

Using (86), (90), (89), and (84), we conclude that

$$
\begin{aligned}
N_{A}^{B}(a) & =\{0\} \times \mathbb{R}_{+} \\
& \varsubsetneqq\left(\{0\} \times \mathbb{R}_{+}\right) \cup\left(\mathbb{R}_{+} \times\{0\}\right)=\varlimsup_{\substack{x \rightarrow a \\
x \in A}}\left(\operatorname{cone}(B-x) \cap N_{A}^{\text {prox }}(x)\right) \\
& \varsubsetneqq(\{0\} \times \mathbb{R}) \cup(\mathbb{R} \times\{0\})=\left(\varlimsup_{\substack{x \rightarrow a \\
x \in A}} \operatorname{cone}(B-x)\right) \cap N_{A}(a) .
\end{aligned}
$$

Therefore, both inclusions in (61) are strict; however, $A$ is not convex and (62) does not hold (see (89)).

Finally, we provide an example where the inclusion (61b) is strict while the inclusion (61c) is an equality. 
Example 5.10 Suppose that $X=\mathbb{R}^{2}$, let $A:=\operatorname{cone}\{(1,0),(0,1)\}, B:=\left\{\left(y_{1}, y_{2}\right) \mid y_{1}+y_{2}=1\right\}$, and $a:=(0,0)$. Let $x=\left(x_{1}, x_{2}\right) \in A$ be sufficiently close to $a$. We compute

$$
\begin{gathered}
\text { cone }(B-x)=\left\{\left(y_{1}, y_{2}\right) \mid y_{1}+y_{2}>0\right\} \cup\{(0,0)\} \\
N_{A}^{\text {prox }}(x)= \begin{cases}\{0\} \times \mathbb{R}, & \text { if } x_{1}>0 \text { and } x_{2}=0 \\
\mathbb{R} \times\{0\}, & \text { if } x_{1}=0 \text { and } x_{2}>0 \\
\mathbb{R}_{-}^{2}, & \text { if } x_{1}=x_{2}=0\end{cases} \\
\widehat{N}_{A}^{B}(x)=\{(0,0)\} .
\end{gathered}
$$

Furthermore, Example 5.8 (see (76)) implies that $N_{A}(a)=\mathbb{R}_{-}^{2} \cup(\{0\} \times \mathbb{R}) \cup(\mathbb{R} \times\{0\})$. We thus deduce that

$$
\begin{aligned}
N_{A}^{B}(a) & =\{(0,0)\} \\
& \varsubsetneqq\left(\{0\} \times \mathbb{R}_{+}\right) \cup\left(\mathbb{R}_{+} \times\{0\}\right)=\varlimsup_{\substack{x \rightarrow a \\
x \in A}}\left(\operatorname{cone}(B-x) \cap N_{A}^{\text {prox }}(x)\right) \\
& =\left(\{0\} \times \mathbb{R}_{+}\right) \cup\left(\mathbb{R}_{+} \times\{0\}\right)=\left(\varlimsup_{\substack{x \rightarrow a \\
x \in A}} \operatorname{cone}(B-x)\right) \cap N_{A}(a) .
\end{aligned}
$$

Therefore, the inclusion (61b) is strict while the inclusion (61c) is an equality.

\section{Constraint qualification conditions and numbers}

Utilizing restricted normal cones, we introduce in this section the notions of CQ-number, joint-CQnumber, CQ condition, and joint-CQ condition, where CQ stands for "constraint qualification".

\section{$C Q$ and joint-CQ numbers}

Definition 6.1 (CQ-number) Let $A, \widetilde{A}, B, \widetilde{B}$, be nonempty subsets of $X$, let $c \in X$, and let $\delta \in \mathbb{R}_{++}$. The CQ-number at $c$ associated with $(A, \widetilde{A}, B, \widetilde{B})$ and $\delta$ is

$$
\theta_{\delta}:=\theta_{\delta}(A, \widetilde{A}, B, \widetilde{B}):=\sup \left\{\begin{array}{ll}
\langle u, v\rangle \mid \begin{array}{l}
u \in \widehat{N}_{A}^{\widetilde{B}}(a), v \in-\widehat{N}_{B}^{\widetilde{A}}(b),\|u\| \leq 1,\|v\| \leq 1, \\
\|a-c\| \leq \delta,\|b-c\| \leq \delta .
\end{array}
\end{array}\right\} .
$$

The limiting CQ-number at c associated with $(A, \widetilde{A}, B, \widetilde{B})$ is

$$
\bar{\theta}:=\bar{\theta}(A, \widetilde{A}, B, \widetilde{B}):=\lim _{\delta \downarrow 0} \theta_{\delta}(A, \widetilde{A}, B, \widetilde{B}) .
$$

Clearly,

$$
\theta_{\delta}(A, \widetilde{A}, B, \widetilde{B})=\theta_{\delta}(B, \widetilde{B}, A, \widetilde{A}) \quad \text { and } \quad \bar{\theta}(A, \widetilde{A}, B, \widetilde{B})=\bar{\theta}(B, \widetilde{B}, A, \widetilde{A})
$$


Note that, $\delta \mapsto \theta_{\delta}$ is increasing; this makes $\bar{\theta}$ well defined. Furthermore, since 0 belongs to nonempty $B$-restricted proximal normal cones and because of the Cauchy-Schwarz inequality, we have

$$
c \in \bar{A} \cap \bar{B} \text { and } 0<\delta_{1}<\delta_{2} \Rightarrow 0 \leq \bar{\theta} \leq \theta_{\delta_{1}} \leq \theta_{\delta_{2}} \leq 1 \text {, }
$$

while $\theta_{\delta}$, and hence $\bar{\theta}$, is equal to $-\infty$ if $c \notin \bar{A} \cap \bar{B}$ and $\delta$ is sufficiently small (using the fact that $\sup \varnothing=-\infty)$. Using Proposition 2.7(ii)\&(vi), we see that

$$
\widetilde{A} \subseteq A^{\prime} \text { and } \widetilde{B} \subseteq B^{\prime} \quad \Rightarrow \quad \theta_{\delta}(A, \widetilde{A}, B, \widetilde{B}) \leq \theta_{\delta}\left(A, A^{\prime}, B, B^{\prime}\right)
$$

and, for every $x \in X$,

$$
\theta_{\delta}(A, \widetilde{A}, B, \widetilde{B}) \text { at } c=\theta_{\delta}(A-x, \widetilde{A}-x, B-x, \widetilde{B}-x) \text { at } c-x \text {. }
$$

To deal with unions, it is convenient to extend this notion as follows.

Definition 6.2 (joint-CQ-number) Let $\mathcal{A}:=\left(A_{i}\right)_{i \in I}, \widetilde{\mathcal{A}}:=\left(\widetilde{A}_{i}\right)_{i \in I}, \mathcal{B}:=\left(B_{j}\right)_{j \in J}, \widetilde{\mathcal{B}}:=\left(\widetilde{B}_{j}\right)_{j \in J}$ be nontrivial collections ${ }^{1}$ of nonempty subsets of $X$, let $c \in X$, and let $\delta \in \mathbb{R}_{++}$. The joint-CQ-number at $c$ associated with $(\mathcal{A}, \widetilde{\mathcal{A}}, \mathcal{B}, \widetilde{\mathcal{B}})$ and $\delta$ is

$$
\theta_{\delta}=\theta_{\delta}(\mathcal{A}, \widetilde{\mathcal{A}}, \mathcal{B}, \widetilde{\mathcal{B}}):=\sup _{(i, j) \in I \times J} \theta_{\delta}\left(A_{i}, \widetilde{A}_{i}, B_{j}, \widetilde{B}_{j}\right)
$$

and the limiting joint-CQ-number at c associated with $(\mathcal{A}, \widetilde{\mathcal{A}}, \mathcal{B}, \widetilde{\mathcal{B}})$ is

$$
\bar{\theta}=\bar{\theta}(\mathcal{A}, \widetilde{\mathcal{A}}, \mathcal{B}, \widetilde{\mathcal{B}}):=\lim _{\delta \downarrow 0} \theta_{\delta}(\mathcal{A}, \widetilde{\mathcal{A}}, \mathcal{B}, \widetilde{\mathcal{B}}) .
$$

For convenience, we will simply write $\theta_{\delta}, \bar{\theta}$ and omit the possible arguments $(A, \widetilde{A}, B, \widetilde{B})$ and $(\mathcal{A}, \widetilde{\mathcal{A}}, \mathcal{B}, \widetilde{\mathcal{B}})$ when there is no cause for confusion. If $I$ and $J$ are singletons, then the notions of $\mathrm{CQ}$-number and joint-CQ-number coincide. Also observe that

$$
c \in \bigcup_{i \in I} A_{i} \cap \bigcup_{j \in J} B_{j} \Rightarrow\left(\forall \delta \in \mathbb{R}_{++}\right) \quad 0 \leq \bar{\theta} \leq \theta_{\delta} \leq 1
$$

while $\bar{\theta}=\theta_{\delta}=-\infty$ when $\delta>0$ is sufficiently small and $c$ does not belong to both $\overline{\bigcup_{i \in I} A_{i}}$ and $\overline{\bigcup_{j \in J} B_{j}}$. Furthermore, the joint-CQ-number (and hence the limiting joint-CQ-number as well) really depends only on those sets $A_{i}$ and $B_{j}$ for which $c \in \overline{A_{i}} \cap \overline{B_{j}}$.

To illustrate this notion, let us compute the CQ-number of two lines. The formula provided is the cosine of the angle between the two lines - as we shall see in Theorem 7.12 below, this happens actually for all linear subspaces although then the angle must be defined appropriately and the proof is more involved.

\footnotetext{
${ }^{1}$ The collection $\left(A_{i}\right)_{i \in I}$ is said to be nontrivial if $I \neq \varnothing$.
} 
Proposition 6.3 (CQ-number of two distinct lines through the origin) Suppose that $w_{a}$ and $w_{b}$ are two vectors in $X$ such that $\left\|w_{a}\right\|=\left\|w_{b}\right\|=1$. Let $A:=\mathbb{R} w_{a}, B:=\mathbb{R} w_{b}$, and $\delta \in \mathbb{R}_{++}$. Assume that $A \cap B=\{0\}$. Then the CQ-number at 0 is

$$
\theta_{\delta}(A, A, B, B)=\left|\left\langle w_{a}, w_{b}\right\rangle\right|
$$

Proof. Set $s:=\left\langle w_{a}, w_{b}\right\rangle$.

Assume first that $s \neq 0$. Let $a=\alpha w_{a} \in A$ and $b=\beta w_{b} \in B$. Then $P_{A}^{-1}(a)-a=N_{A}(a)=\left\{w_{a}\right\}^{\perp}$; considering $(B-a) \cap\left\{w_{a}\right\}^{\perp}$ leads to $\beta s=\alpha$. Hence $\left(P_{A}^{-1}(a)-a\right) \cap(B-a)=\beta w_{b}-\alpha w_{a}$ and

$$
\widehat{N}_{A}^{B}(a)=\text { cone }\left(\alpha s^{-1} w_{b}-\alpha w_{a}\right) .
$$

Similarly,

$$
-\widehat{N}_{B}^{A}(b)=\text { cone }\left(\beta w_{b}-\beta s^{-1} w_{a}\right) .
$$

Now set $u:=\alpha s^{-1} w_{b}-\alpha w_{a} \in \widehat{N}_{A}^{B}(a)$ and $v:=\beta w_{b}-\beta s^{-1} w_{a} \in-\widehat{N}_{B}^{A}(b)$. One computes

$$
\|u\|=\frac{|\alpha| \sqrt{1-s^{2}}}{|s|},\|v\|=\frac{|\beta| \sqrt{1-s^{2}}}{|s|} \text {, and }\langle u, v\rangle=\frac{\alpha \beta\left(1-s^{2}\right)}{s} \text {. }
$$

Hence

$$
\frac{\langle u, v\rangle}{\|u\| \cdot\|v\|}=\operatorname{sgn}(\alpha) \operatorname{sgn}(\beta) s
$$

Choosing $\alpha$ and $\beta$ in $\{-1,1\}$ appropriately, we arrange for $\langle u, v\rangle /(\|u\| \cdot\|v\|)=|s|$, as claimed.

Now assume that $s=0$. Arguing similarly, we see that

$$
(\forall a \in A) \quad \widehat{N}_{A}^{B}(a)=\left\{\begin{array}{ll}
\{0\}, & \text { if } a \neq 0 ; \\
B, & \text { if } a=0,
\end{array} \quad \text { and } \quad(\forall b \in B) \quad \widehat{N}_{B}^{A}(b)= \begin{cases}\{0\}, & \text { if } b \neq 0 \\
A, & \text { if } b=0\end{cases}\right.
$$

This leads to $\theta_{\delta}(A, A, B, B)=0=|s|$, again as claimed.

Let $\mathcal{A}:=\left(A_{i}\right)_{i \in I}, \widetilde{\mathcal{A}}:=\left(\widetilde{A}_{i}\right)_{i \in I}, \mathcal{B}:=\left(B_{j}\right)_{j \in J}$ and $\widetilde{\mathcal{B}}:=\left(\widetilde{B}_{j}\right)_{j \in J}$ be nontrivial collections of nonempty closed subsets of $X$ and let $\delta \in \mathbb{R}_{++}$. Set $A:=\bigcup_{i \in I} A_{i}, \widetilde{A}:=\bigcup_{i \in I} \widetilde{A}_{i}, B:=\bigcup_{j \in J} B_{j}$, $\widetilde{B}:=\bigcup_{j \in J} \widetilde{B}_{j}$, and suppose that $c \in A \cap B$. It is interesting to compare the joint-CQ-number of collections, i.e., $\theta_{\delta}(\mathcal{A}, \widetilde{\mathcal{A}}, \mathcal{B}, \widetilde{\mathcal{B}})$, to the $C Q$-number of the unions, i.e., $\theta_{\delta}(A, \widetilde{A}, B, \widetilde{B})$. We shall see in the following two examples that neither of them is smaller than the other; in fact, one of them can be equal to 1 while the other is strictly less than 1 . These examples will illustrate the independence of the two types of CQ-numbers (for the collection and for the union). In some cases, such as Example 6.4, it is beneficial to work with a suitable partition to obtain a CQ-number that is less than one, which in turn is very desirable in applications (see [3] and [4]). 
Example 6.4 (joint-CQ-number $<\mathrm{CQ}$-number of the unions) Suppose that $X=\mathbb{R}^{3}$, let $I:=$ $J:=\{1,2\}, A_{1}:=\mathbb{R}(0,1,0), A_{2}:=\mathbb{R}(2,0,-1), B_{1}:=\mathbb{R}(0,1,1), B_{2}:=\mathbb{R}(1,0,0), c:=(0,0,0)$, and let $\delta>0$. Furthermore, set $\mathcal{A}:=\left(A_{i}\right)_{i \in I}, \mathcal{B}:=\left(B_{j}\right)_{j \in J}, A:=A_{1} \cup A_{2}$, and $B:=B_{1} \cup B_{2}$. Then

$$
\theta_{\delta}(\mathcal{A}, \mathcal{A}, \mathcal{B}, \mathcal{B})=\frac{2}{\sqrt{5}}<1=\theta_{\delta}(A, A, B, B)
$$

Proof. Using Proposition 6.3, we compute, for the reference point $c$,

$$
\begin{aligned}
& \theta_{\delta}\left(A_{1}, A_{1}, B_{1}, B_{1}\right)=\left|\left\langle(0,1,0), \frac{1}{\sqrt{2}}(0,1,1)\right\rangle\right|=\frac{1}{\sqrt{2}} \\
& \theta_{\delta}\left(A_{1}, A_{1}, B_{2}, B_{2}\right)=|\langle(0,1,0),(1,0,0)\rangle|=0, \\
& \theta_{\delta}\left(A_{2}, A_{2}, B_{1}, B_{1}\right)=\left|\left\langle\frac{1}{\sqrt{5}}(2,0,-1), \frac{1}{\sqrt{2}}(0,1,1)\right\rangle\right|=\frac{1}{\sqrt{10}} \\
& \theta_{\delta}\left(A_{2}, A_{2}, B_{2}, B_{2}\right)=\mid\left\langle\frac{1}{\sqrt{5}}(2,0,-1),(1,0,0\rangle\right|=\frac{2}{\sqrt{5}} .
\end{aligned}
$$

Hence $\theta_{\delta}(\mathcal{A}, \mathcal{A}, \mathcal{B}, \mathcal{B})=\max _{(i, j) \in I \times J} \theta_{\delta}\left(A_{i}, A_{i}, B_{j}, B_{j}\right)=\frac{2}{\sqrt{5}}<1$.

To estimate the CQ-number of the union, set

$$
a:=(0, \delta, 0) \in A_{1} \subseteq A \text { and } b:=(\delta, 0,0) \in B_{2} \subseteq B .
$$

Note that $\|a-c\|=\|a\|=\delta$ and $\|b-c\|=\|b\|=\delta$. Now define

$$
\widetilde{a}:=(\delta, 0,-\delta / 2) \in A_{2} \subseteq A \text { and } \widetilde{b}:=(0, \delta, \delta) \in B_{1} \subseteq B .
$$

Since $\left\|\widetilde{a}-P_{B_{2}} \tilde{a}\right\|<\left\|\widetilde{a}-P_{B_{1}} \tilde{a}\right\|$ and $P_{B_{2}} \tilde{a}=b$, we have $b=P_{B} \tilde{a}$. Since $\left\|\widetilde{b}-P_{A_{1}} \widetilde{b}\right\|<\left\|\widetilde{b}-P_{A_{2}} \widetilde{b}\right\|$ and $P_{A_{1}} \widetilde{b}=a$, we have $a=P_{A} \widetilde{b}$. Therefore, $\widetilde{b} \in B \cap P_{A}^{-1}(a)$ and $\widetilde{a} \in A \cap P_{B}^{-1}(b)$. It follows that

$$
\begin{aligned}
u & :=\frac{1}{\delta}(\widetilde{b}-a)=(0,0,1) \in \widehat{N}_{A}^{B}(a), \\
v & :=\frac{2}{\delta}(b-\widetilde{a})=(0,0,1) \in-\widehat{N}_{B}^{A}(b) .
\end{aligned}
$$

Since $\|u\|=\|v\|=1$, we obtain $1=\langle u, v\rangle \leq \theta_{\delta}(A, A, B, B) \leq 1$.

Example 6.5 (CQ-number of the unions $<$ joint-CQ-number) Suppose that $X=\mathbb{R}$, let $I:=J:=$ $\{1,2\}, A_{1}:=B_{1}:=\mathbb{R}_{-}, A_{2}:=B_{2}:=\mathbb{R}_{+}, c:=0$, and $\delta>0$. Furthermore, set $\mathcal{A}:=\left(A_{i}\right)_{i \in I}$, $\mathcal{B}:=\left(B_{j}\right)_{j \in I}, A:=A_{1} \cup A_{2}=\mathbb{R}$, and $B:=B_{1} \cup B_{2}=\mathbb{R}$. Then

$$
\theta_{\delta}(A, A, B, B)=0<1=\theta_{\delta}(\mathcal{A}, \mathcal{A}, \mathcal{B}, \mathcal{B})
$$

Proof. Lemma 2.4(viii) implies that $(\forall x \in \mathbb{R}) \widehat{N}_{\mathbb{R}}^{\mathbb{R}}(x)=\{0\}$. Hence $\theta_{\delta}(\mathbb{R}, \mathbb{R}, \mathbb{R}, \mathbb{R})=0$ as claimed. On the other hand, $\widehat{N}_{\mathbb{R}_{+}}^{\mathbb{R}_{-}}(0)=\mathbb{R}_{-}$and $\widehat{N}_{\mathbb{R}_{-}}^{\mathbb{R}_{+}}(0)=\mathbb{R}_{+}$. Hence $\theta_{\delta}\left(\mathbb{R}_{-}, \mathbb{R}_{-}, \mathbb{R}_{+}, \mathbb{R}_{+}\right)=1$ and therefore $\theta_{\delta}(\mathcal{A}, \mathcal{A}, \mathcal{B}, \mathcal{B})=1$ as well. 


\section{$\mathrm{CQ}$ and joint-CQ conditions}

Definition 6.6 (CQ and joint-CQ conditions) Let $c \in X$.

(i) Let $A, \widetilde{A}, B$ and $\widetilde{B}$ be nonempty subsets of $X$. Then the $(A, \widetilde{A}, B, \widetilde{B})-\mathrm{CQ}$ condition holds at $c$ if

$$
N_{A}^{\widetilde{B}}(c) \cap\left(-N_{B}^{\widetilde{A}}(c)\right) \subseteq\{0\} .
$$

(ii) Let $\mathcal{A}:=\left(A_{i}\right)_{i \in I}, \widetilde{\mathcal{A}}:=\left(\widetilde{A}_{i}\right)_{i \in I}, \mathcal{B}:=\left(B_{j}\right)_{j \in J}$ and $\widetilde{\mathcal{B}}:=\left(\widetilde{B}_{j}\right)_{j \in J}$ be nontrivial collections of nonempty subsets of $X$. Then the $(\mathcal{A}, \widetilde{\mathcal{A}}, \mathcal{B}, \widetilde{\mathcal{B}})$-joint-CQ condition holds at $c$ if for every $(i, j) \in$ $I \times J$, the $\left(A_{i}, \widetilde{A}_{i}, B_{j}, \widetilde{B}_{j}\right)-C Q$ condition holds at $c$, i.e.,

$$
(\forall(i, j) \in I \times J) \quad N_{A_{i}}^{\widetilde{B}_{j}}(c) \cap\left(-N_{B_{j}}^{\widetilde{A}_{i}}(c)\right) \subseteq\{0\} .
$$

In view of the definitions, the key case to consider is when $c \in A \cap B$ (or when $c \in A_{i} \cap B_{j}$ in the joint-CQ case). The CQ-number is based on the behavior of the restricted proximal normal cone in a neighborhood of the point under consideration - a related notion is that of the exact CQ-number, where we consider the restricted normal cone at the point instead of nearby restricted proximal normal cones.

Definition 6.7 (exact CQ-number and exact joint-CQ-number) Let $c \in X$.

(i) Let $A, \widetilde{A}, B$ and $\widetilde{B}$ be nonempty subsets of $X$. The exact CQ-number at $c$ associated with $(A, \widetilde{A}, B, \widetilde{B})$ is ${ }^{2}$

$$
\bar{\alpha}:=\bar{\alpha}(A, \widetilde{A}, B, \widetilde{B}):=\sup \left\{\langle u, v\rangle \mid u \in N_{A}^{\widetilde{B}}(c), v \in-N_{B}^{\widetilde{A}}(c),\|u\| \leq 1,\|v\| \leq 1\right\} .
$$

(ii) Let $\mathcal{A}:=\left(A_{i}\right)_{i \in I}, \widetilde{\mathcal{A}}:=\left(\widetilde{A}_{i}\right)_{i \in I}, \mathcal{B}:=\left(B_{j}\right)_{j \in J}$ and $\widetilde{\mathcal{B}}:=\left(\widetilde{B}_{j}\right)_{j \in J}$ be nontrivial collections of nonempty subsets of $X$. The exact joint-CQ-number at c associated with $(\mathcal{A}, \mathcal{B}, \widetilde{\mathcal{A}}, \widetilde{\mathcal{B}})$ is

$$
\bar{\alpha}:=\bar{\alpha}(\mathcal{A}, \widetilde{\mathcal{A}}, \mathcal{B}, \widetilde{\mathcal{B}}):=\sup _{(i, j) \in I \times J} \bar{\alpha}\left(A_{i}, \widetilde{A}_{i}, B_{j}, \widetilde{B}_{j}\right) .
$$

The next result relates the various condition numbers defined above.

Theorem 6.8 Let $\mathcal{A}:=\left(A_{i}\right)_{i \in I}, \widetilde{\mathcal{A}}:=\left(\widetilde{A}_{i}\right)_{i \in I}, \mathcal{B}:=\left(B_{j}\right)_{j \in J}$ and $\widetilde{\mathcal{B}}:=\left(\widetilde{B}_{j}\right)_{j \in J}$ be nontrivial collections of nonempty subsets of $X$. Set $A:=\bigcup_{i \in I} A_{i}$ and $B:=\bigcup_{j \in J} B_{j}$, and suppose that $c \in A \cap B$. Denote the exact joint-CQ-number at $c$ associated with $(\mathcal{A}, \widetilde{\mathcal{A}}, \mathcal{B}, \widetilde{\mathcal{B}})$ by $\bar{\alpha}$ (see $(118)$ ), the joint-CQ-number at $c$ associated with $(\mathcal{A}, \widetilde{\mathcal{A}}, \mathcal{B}, \widetilde{\mathcal{B}})$ and $\delta>0$ by $\theta_{\delta}$ (see (100)), and the limiting joint-CQ-number at c associated with $(\mathcal{A}, \widetilde{\mathcal{A}}, \mathcal{B}, \widetilde{\mathcal{B}})$ by $\bar{\theta}$ (see (101)). Then the following hold:

\footnotetext{
${ }^{2}$ Note that if $c \notin A \cap B$, then $\bar{\alpha}=\sup \varnothing=-\infty$.
} 
(i) If $\bar{\alpha}<1$, then the $(\mathcal{A}, \widetilde{\mathcal{A}}, \mathcal{B}, \widetilde{\mathcal{B}})-C Q$ condition holds at $c$.

(ii) $\bar{\alpha} \leq \theta_{\delta}$.

(iii) $\bar{\alpha} \leq \bar{\theta}$.

Now assume in addition that I and J are finite. Then the following hold:

(iv) $\bar{\alpha}=\bar{\theta}$.

(v) The $(\mathcal{A}, \widetilde{\mathcal{A}}, \mathcal{B}, \widetilde{\mathcal{B}})$-joint-CQ condition holds at $\mathrm{c}$ if and only if $\bar{\alpha}=\bar{\theta}<1$.

Proof. (i): Suppose that $\bar{\alpha}<1$. The condition for equality in the Cauchy-Schwarz inequality implies that for all $(i, j) \in I \times J$, the intersection $N_{A_{i}}^{\widetilde{B}_{j}}(c) \cap\left(-N_{B_{j}}^{\widetilde{A}_{i}}(c)\right)$ is either empty or $\{0\}$. In view of Definition 6.6 , we see that the $(\mathcal{A}, \widetilde{\mathcal{A}}, \mathcal{B}, \widetilde{\mathcal{B}})$-joint-CQ holds at $c$.

(ii): Let $(i, j) \in I \times J$. Take $u \in N_{A_{i}}^{\widetilde{B}_{j}}(c)$ and $v \in-N_{B_{j}}^{\widetilde{A}_{i}}(c)$ such that $\|u\| \leq 1$ and $\|v\| \leq 1$. Then, by definition of the restricted normal cone, there exist sequences $\left(a_{n}\right)_{n \in \mathbb{N}}$ in $A_{i},\left(b_{n}\right)_{n \in \mathbb{N}}$ in $B_{j}$, $\left(u_{n}\right)_{n \in \mathbb{N}}$ and $\left(v_{n}\right)_{n \in \mathbb{N}}$ in $X$ such that $a_{n} \rightarrow c, b_{n} \rightarrow c, u_{n} \rightarrow u, v_{n} \rightarrow v$, and $(\forall n \in \mathbb{N}) u_{n} \in \widehat{N}_{A_{i}}^{\widetilde{B}_{j}}\left(a_{n}\right)$ and $v_{n} \in-\widehat{N}_{B_{j}}^{\widetilde{A}_{i}}\left(b_{n}\right)$. Note that since $\delta>0$, eventually $a_{n}$ and $b_{n}$ lie in ball $(c ; \delta)$; consequently, $\left\langle u_{n}, v_{n}\right\rangle \leq \theta_{\delta}\left(A_{i}, \widetilde{A}_{i}, B_{j}, \widetilde{B}_{j}\right)$. Taking the limit as $n \rightarrow+\infty$, we obtain $\langle u, v\rangle \leq \theta_{\delta}\left(A_{i}, \widetilde{A}_{i}, B_{j}, \widetilde{B}_{j}\right) \leq \theta_{\delta}$. Now taking the supremum over suitable $u$ and $v$, followed by taking the supremum over $(i, j)$, we conclude that $\bar{\alpha} \leq \theta_{\delta}$.

(iii): This is clear from (ii) and (101).

(iv): Let $\left(\delta_{n}\right)_{n \in \mathbb{N}}$ be a sequence in $\mathbb{R}_{++}$such that $\delta_{n} \rightarrow 0$. Then for every $n \in \mathbb{N}$, there exist

$$
i_{n} \in I, j_{n} \in J, a_{n} \in A_{i_{n}}, b_{n} \in B_{j_{n}}, u_{n} \in \widehat{N}_{A_{i_{n}}}^{\widetilde{B}_{j_{n}}}\left(a_{n}\right), v_{n} \in-\widehat{N}_{B_{j_{n}}}^{\widetilde{A}_{i_{n}}}\left(b_{n}\right)
$$

such that

$$
\left\|a_{n}-c\right\| \leq \delta_{n},\left\|b_{n}-c\right\| \leq \delta_{n},\left\|u_{n}\right\| \leq 1,\left\|v_{n}\right\| \leq 1, \text { and }\left\langle u_{n}, v_{n}\right\rangle>\theta_{\delta_{n}}-\delta_{n} .
$$

Since $I$ and $J$ are finite, and after passing to a subsequence and relabeling if necessary, we can and do assume that there exists $(i, j) \in I \times J$ such that $u_{n} \rightarrow u \in N_{A_{i}}^{\widetilde{B}_{j}}(c)$ and $v_{n} \rightarrow v \in-N_{B_{j}}^{\widetilde{A}_{i}}(c)$. Hence $\bar{\theta} \leftarrow \theta_{\delta_{n}}-\delta_{n}<\left\langle u_{n}, v_{n}\right\rangle \rightarrow\langle u, v\rangle \leq \bar{\alpha}$. Hence $\bar{\theta} \leq \bar{\alpha}$. On the other hand, $\bar{\alpha} \leq \bar{\theta}$ by (iii). Altogether, $\bar{\alpha}=\bar{\theta}$.

(v): " $\Rightarrow$ ": Let $(i, j) \in I \times J$. If $c \notin A_{i} \cap B_{j}$, then $\bar{\alpha}\left(A_{i}, \widetilde{A}_{i}, B_{j}, \widetilde{B}_{j}\right)=-\infty$. Now assume that $c \in A_{i} \cap B_{j}$. Since the $(\mathcal{A}, \widetilde{\mathcal{A}}, \mathcal{B}, \widetilde{\mathcal{B}})$-joint-CQ condition holds, we have $N_{A_{i}}^{\widetilde{B}_{j}}(c) \cap-N_{B_{j}}^{\widetilde{A}_{i}}(c)=\{0\}$. By Cauchy-Schwarz,

$$
\bar{\alpha}\left(A_{i}, \widetilde{A}_{i}, B_{j}, \widetilde{B}_{j}\right)=\sup \left\{\langle u, v\rangle \mid u \in N_{A_{i}}^{\widetilde{B}_{j}}(c), v \in-N_{B_{j}}^{\widetilde{A}_{i}}(c),\|u\| \leq 1,\|v\| \leq 1\right\}<1 .
$$


Since $I$ and $J$ are finite and because of (iv), we deduce that $\bar{\theta}=\bar{\alpha}<1$.

"Æ": Combine (i) with (iv).

\section{CQ conditions and $C Q$ numbers: examples}

In this section, we provide further results and examples illustrating CQ conditions and CQ numbers.

First, let us note that the assumption that the sets of indices be finite in Theorem 6.8(iv) is essential:

Example 7.1 $(\bar{\alpha}<\bar{\theta})$ Suppose that $X=\mathbb{R}^{2}$, let $\Gamma \subseteq \mathbb{R}_{++}$be such that $\sup \Gamma=+\infty$, set $(\forall \gamma \in \Gamma)$ $A_{\gamma}:=\operatorname{epi}\left(\frac{1}{2} \gamma|\cdot|^{2}\right), B:=\mathbb{R}_{+} \times \mathbb{R}, \mathcal{A}:=\left(A_{\gamma}\right)_{\gamma \in \Gamma}, \widetilde{\mathcal{A}}:=(X)_{\gamma \in \Gamma}, \mathcal{B}:=(B), \widetilde{\mathcal{B}}:=(X)$, and $c:=(0,0)$. Denote the exact joint-CQ-number at $c$ associated with $(\mathcal{A}, \widetilde{\mathcal{A}}, \mathcal{B}, \widetilde{\mathcal{B}})$ by $\bar{\alpha}$ (see (118)), the joint-CQ-number at $c$ associated with $(\mathcal{A}, \widetilde{\mathcal{A}}, \mathcal{B}, \widetilde{\mathcal{B}})$ and $\delta>0$ by $\theta_{\delta}$ (see (100)), and the limiting joint-CQ-number at $c$ associated with $(\mathcal{A}, \widetilde{\mathcal{A}}, \mathcal{B}, \widetilde{\mathcal{B}})$ by $\bar{\theta}$ (see (101)). Then

$$
\bar{\alpha}=0<1=\theta_{\delta}=\bar{\theta} .
$$

Proof. Let $\gamma \in \Gamma$ and pick $x>0$ such that $a:=\left(x, \frac{1}{2} \gamma x^{2}\right) \in A_{\gamma}$ satisfies $\|a\|=\|a-c\|=\delta$, i.e., $x>0$ and

$$
\gamma^{2} x^{2}=2\left(\sqrt{1+\gamma^{2} \delta^{2}}-1\right) \rightarrow+\infty \quad \text { as } \gamma \rightarrow+\infty \text { in } \Gamma
$$

Hence

$$
\gamma x \rightarrow+\infty, \quad \text { as } \gamma \rightarrow+\infty \text { in } \Gamma .
$$

Since $A_{\gamma}$ is closed and convex, it follows from Lemma 2.4(vii) that

$$
u:=\frac{(\gamma x,-1)}{\sqrt{\gamma^{2} x^{2}+1}} \in \mathbb{R}_{+}(\gamma x,-1)=N_{A_{\gamma}}^{\mathrm{conv}}(a)=\widehat{N}_{A_{\gamma}}^{X}(a)=N_{A_{\gamma}}^{X}(a)=N_{A_{\gamma}}(a)
$$

Furthermore, $v:=(1,0) \in-\left(\mathbb{R}_{-} \times\{0\}\right)=-\widehat{N}_{B}^{X}(c)=-N_{B}^{X}(c)=-N_{B}(c),\|u\|=\|v\|=1$, and, in view of (124),

$$
\begin{aligned}
1 & \geq \theta_{\delta} \geq \theta_{\delta}\left(A_{\gamma}, X, B, X\right) \geq\langle u, v\rangle=\frac{\gamma x}{\sqrt{\gamma^{2} x^{2}+1}} \\
& \rightarrow 1 \quad \text { as } \gamma \rightarrow+\infty \text { in } \Gamma .
\end{aligned}
$$

Thus $\theta_{\delta}=1$, which implies that $\bar{\theta}=1$. Finally, $N_{A_{\gamma}}(c)=\left(\{0\} \times \mathbb{R}_{-}\right) \perp\left(\mathbb{R}_{+} \times\{0\}\right)=-N_{B}(c)$, which shows that $\bar{\alpha}=0$. 
For the eventual application of these results to the method of alternating projections, the condition $\bar{\alpha}=\bar{\theta}<1$ is critical to ensure linear convergence.

The following example illustrates that the CQ-number can be interpreted as a quantification of the CQ condition.

Example 7.2 (CQ-number quantifies CQ condition) Let $A$ and $B$ be subsets of $X$, and suppose that $c \in A \cap B$. Let $L$ be an affine subspace of $X$ containing $A \cup B$. Then the following are equivalent:

(i) $N_{A}^{L}(c) \cap\left(-N_{B}^{L}(c)\right)=\{0\}$, i.e., the $(A, L, B, L)$-CQ condition holds at $c$ (see (115)).

(ii) $N_{A}(c) \cap\left(-N_{B}(c)\right) \cap(L-c)=\{0\}$.

(iii) $\bar{\theta}<1$, where $\bar{\theta}$ is the limiting CQ-number at $c$ associated with $(A, L, B, L)$ (see (95)).

Proof. The identity (25d) of Theorem 3.5 yields $N_{A}^{L}(c)=N_{A}(c) \cap(L-c)$ and $N_{B}^{L}(c)=N_{B}(c) \cap$ $(L-c)$. Hence

$$
N_{A}^{L}(c) \cap\left(-N_{B}^{L}(c)\right)=N_{A}(c) \cap\left(-N_{B}(c)\right) \cap(L-c),
$$

and the equivalence of (i) and (ii) is now clear. Finally, Theorem 6.8(iv)\&(v) yields the equivalence of (i) and (iii).

Depending on the choice of the restricting sets $\widetilde{A}$ and $\widetilde{B}$, the $(A, \widetilde{A}, B, \widetilde{B})-\mathrm{CQ}$ condition may either hold or fail:

Example 7.3 (CQ condition depends on restricting sets) Suppose that $X=\mathbb{R}^{2}$, and set $A:=$ $\operatorname{epi}(|\cdot|), B:=\mathbb{R} \times\{0\}$, and $c:=(0,0)$. Then we readily verify that $N_{A}(c)=N_{A}^{X}(c)=-A$, $N_{A}^{B}(c)=-$ bdry $A, N_{B}(c)=N_{B}^{X}(c)=\{0\} \times \mathbb{R}$, and $N_{B}^{A}(c)=\{0\} \times \mathbb{R}_{+}$. Consequently,

$$
N_{A}^{X}(c) \cap\left(-N_{B}^{X}(c)\right)=\{0\} \times \mathbb{R}_{-} \text {while } N_{A}^{B}(c) \cap\left(-N_{B}^{A}(c)\right)=\{(0,0)\} .
$$

Therefore, the $(A, A, B, B)-C Q$ condition holds, yet the $(A, X, B, X)-C Q$ condition fails.

For two spheres, it is possible to quantify the convergence of $\theta_{\delta}$ to $\bar{\delta}=\bar{\alpha}$ :

Proposition 7.4 (CQ-numbers of two spheres) Let $z_{1}$ and $z_{2}$ be in $X$, let $\rho_{1}$ and $\rho_{2}$ be in $\mathbb{R}_{++}$, set $S_{1}:=\operatorname{sphere}\left(z_{1} ; \rho_{1}\right)$ and $S_{2}:=\operatorname{sphere}\left(z_{2} ; \rho_{2}\right)$ and assume that $c \in S_{1} \cap S_{2}$. Denote the limiting $C Q$-number at $c$ associated with $\left(S_{1}, X, S_{2}, X\right)$ by $\bar{\theta}$ (see Definition 6.1), and the exact CQ-number at $c$ associated with $\left(S_{1}, X, S_{2}, X\right)$ by $\bar{\alpha}$ (see Definition 6.7). Then the following hold:

(i) $\bar{\theta}=\bar{\alpha}=\frac{\left|\left\langle z_{1}-c, z_{2}-c\right\rangle\right|}{\rho_{1} \rho_{2}}$.

(ii) $\bar{\alpha}<1$ unless the spheres are identical or intersect only at $c$. 
Now assume that $\bar{\alpha}<1$, let $\varepsilon \in \mathbb{R}_{++}$, and set $\delta:=\left(\sqrt{\left(\rho_{1}+\rho_{2}\right)^{2}+4 \rho_{1} \rho_{2} \varepsilon}-\left(\rho_{1}+\rho_{2}\right)\right) / 2>0$. Then

$$
\bar{\alpha} \leq \theta_{\delta} \leq \bar{\alpha}+\varepsilon,
$$

where $\theta_{\delta}$ is the CQ-number at c associated with $\left(S_{1}, X, S_{2}, X\right)$ (see Definition 6.1).

Proof. (i): This follows from Theorem 6.8(iv) and Example 2.6.

(ii): Combine (i) with the characterization of equality in the Cauchy-Schwarz inequality.

Let us now establish (129). By Theorem 6.8(ii), we have $\bar{\alpha} \leq \theta_{\delta}$. Let $s_{1} \in S_{1}$ be such that $\left\|s_{1}-c\right\| \leq \delta$, let $u_{1} \in \widehat{N}_{S_{1}}^{X}\left(s_{1}\right)$ be such that $\left\|u_{1}\right\|=1$, let $s_{2} \in S_{2}$ be such that $\left\|s_{2}-c\right\| \leq \delta$, and let $u_{2} \in \widehat{N}_{S_{2}}^{X}\left(s_{2}\right)$ be such that $\left\|u_{2}\right\|=1$. By Example 2.6,

$$
u_{1}= \pm \frac{s_{1}-z_{1}}{\left\|s_{1}-z_{1}\right\|}= \pm \frac{s_{1}-z_{1}}{\rho_{1}} \quad \text { and } \quad u_{2}= \pm \frac{s_{2}-z_{2}}{\left\|s_{2}-z_{2}\right\|}= \pm \frac{s_{2}-z_{2}}{\rho_{2}}
$$

Hence

$$
\begin{aligned}
\rho_{1} \rho_{2}\left\langle u_{1}, u_{2}\right\rangle \leq & \left|\left\langle s_{1}-z_{1}, s_{2}-z_{2}\right\rangle\right| \\
= & \left|\left\langle\left(s_{1}-c\right)+\left(c-z_{1}\right),\left(s_{2}-c\right)+\left(c-z_{2}\right)\right\rangle\right| \\
\leq & \left|\left\langle s_{1}-c, s_{2}-c\right\rangle\right|+\left|\left\langle s_{1}-c, c-z_{2}\right\rangle\right| \\
& \quad+\left|\left\langle c-z_{1}, s_{2}-c\right\rangle\right|+\left|\left\langle c-z_{1}, c-z_{2}\right\rangle\right| \\
\leq & \delta^{2}+\delta\left(\rho_{1}+\rho_{2}\right)+\rho_{1} \rho_{2} \bar{\alpha}
\end{aligned}
$$

and thus, using the definition of $\delta$,

$$
\left\langle u_{1}, u_{2}\right\rangle \leq \bar{\alpha}+\frac{\delta^{2}+\delta\left(\rho_{1}+\rho_{2}\right)}{\rho_{1} \rho_{2}}=\bar{\alpha}+\varepsilon .
$$

Therefore, by the definition of $\theta_{\delta}$, we have $\theta_{\delta} \leq \bar{\alpha}+\varepsilon$.

\section{Two convex sets}

Let us turn to the classical convex setting. We start by noting that well known constraint qualifications are conveniently characterized using our CQ conditions.

Proposition 7.5 (classical convex setting I) Let $A$ and $B$ be nonempty convex subsets of $X$ such that $A \cap B \neq \varnothing$, and set $L=\operatorname{aff}(A \cup B)$. Then the following are equivalent:

(i) ri $A \cap$ ri $B \neq \varnothing$.

(ii) The $(A, L, B, L)-C Q$ condition holds at some point in $A \cap B$.

(iii) The $(A, L, B, L)-C Q$ condition holds at every point in $A \cap B$. 
Proof. This is clear from Theorem 3.13.

Proposition 7.6 (classical convex setting II) Let $A$ and $B$ be nonempty convex subsets of $X$ such that $A \cap B \neq \varnothing$. Then the following are equivalent:

(i) $0 \in \operatorname{int}(B-A)$.

(ii) The $(A, X, B, X)-C Q$ condition holds at some point in $A \cap B$.

(iii) The $(A, X, B, X)-C Q$ condition holds at every point in $A \cap B$.

Proof. This is clear from Corollary 3.14 .

In stark contrast to Proposition 7.5 and 7.6, if the restricting sets are not both equal to $L$ or to $X$, then the CQ-condition may actually depend on the reference point as we shall illustrate now:

Example 7.7 (CQ condition depends on the reference point) Suppose that $X=\mathbb{R}^{2}$, and let $f: \mathbb{R} \rightarrow \mathbb{R}: x \mapsto(\max \{0, x\})^{2}$, which is a continuous convex function. Set $A:=$ epi $f$ and $B:=\mathbb{R} \times\{0\}$, which are closed convex subsets of $X$. Consider first the point $c:=(-1,0) \in A \cap B$. Then $N_{A}^{B}(c)=\{(0,0)\}$ and $N_{B}^{A}(c)=\{0\} \times \mathbb{R}_{+}$; hence,

$$
N_{A}^{B}(c) \cap\left(-N_{B}^{A}(c)\right)=\{(0,0)\},
$$

i.e., the $(A, A, B, B)-C Q$ condition holds at $c$. On the other hand, consider now $d:=(0,0) \in A \cap B$. Then $N_{A}^{B}(d)=\{0\} \times \mathbb{R}_{-}$and $N_{B}^{A}(d)=\{0\} \times \mathbb{R}_{+} ;$thus,

$$
N_{A}^{B}(d) \cap\left(-N_{B}^{A}(d)\right)=\{0\} \times \mathbb{R}_{-},
$$

i.e., the $(A, A, B, B)-\mathrm{CQ}$ condition fails at $d$.

\section{Two linear (or intersecting affine) subspaces}

We specialize further to two linear subspaces of X. A pleasing connection between CQ-number and the angle between two linear subspaces will be revealed. But first we provide some auxiliary results.

Proposition 7.8 Let $A$ and $B$ be linear subspaces of $X$, and let $\delta \in \mathbb{R}_{++}$. Then

$$
\bigcup_{a \in A \cap\left(B+A^{\perp}\right) \cap \operatorname{ball}(0 ; \delta)} \widehat{N}_{A}^{B}(a)=\bigcup_{a \in A \cap \operatorname{ball}(0 ; \delta)} \widehat{N}_{A}^{B}(a)=\bigcup_{a \in A} \widehat{N}_{A}^{B}(a)=A^{\perp} \cap(A+B) .
$$

Proof. Let $a \in A$. Then $P_{A}^{-1}(a)=a+A^{\perp}$ and hence $P_{A}^{-1}(a)-a=A^{\perp}$. If $B \cap\left(a+A^{\perp}\right)=\varnothing$, then $\widehat{N}_{A}^{B}(a)=\{0\}$. Thus we assume that $B \cap\left(a+A^{\perp}\right) \neq \varnothing$, which is equivalent to $a \in A \cap\left(B+A^{\perp}\right)$. 
Next, by Lemma 2.4(ii), $\widehat{N}_{A}^{B}(a)=A^{\perp} \cap \operatorname{cone}(B-a)$. This implies $\left(\forall \lambda \in \mathbb{R}_{++}\right)$cone $(B-\lambda a)=$ cone $(\lambda(B-a))=\operatorname{cone}(B-a)$. Thus,

$$
\left(\forall \lambda \in \mathbb{R}_{++}\right) \quad \widehat{N}_{A}^{B}(\lambda a)=A^{\perp} \cap \operatorname{cone}(B-\lambda a)=A^{\perp} \cap \operatorname{cone}(B-a)=\widehat{N}_{A}^{B}(a) .
$$

This establishes not only the first two equalities in (135) but also the third because

$$
\begin{aligned}
\bigcup_{a \in A} \widehat{N}_{A}^{B}(a) & =\bigcup_{a \in A}\left(A^{\perp} \cap \operatorname{cone}(B-a)\right)=A^{\perp} \cap \bigcup_{a \in A} \operatorname{cone}(B-a) \\
& =A^{\perp} \cap \operatorname{cone}\left(\bigcup_{a \in A}(B-a)\right)=A^{\perp} \cap \operatorname{cone}(B-A)=A^{\perp} \cap(B-A) \\
& =A^{\perp} \cap(B+A) .
\end{aligned}
$$

The proof is complete.

We now introduce two notions of angles between subspaces; for further information, we highly recommend [8] and [9].

Definition 7.9 Let $A$ and $B$ be linear subspaces of $X$.

(i) (Dixmier angle) [10] The Dixmier angle between $A$ and $B$ is the number in $\left[0, \frac{\pi}{2}\right]$ whose cosine is given by

$$
c_{0}(A, B):=\sup \{|\langle a, b\rangle| \mid a \in A, b \in B,\|a\| \leq 1,\|b\| \leq 1\} .
$$

(ii) (Friedrichs angle) [11] The Friedrichs angle (or simply the angle) between $A$ and $B$ is the number in $\left[0, \frac{\pi}{2}\right]$ whose cosine is given by

$$
\begin{aligned}
c(A, B) & :=c_{0}\left(A \cap(A \cap B)^{\perp}, B \cap(A \cap B)^{\perp}\right) \\
& =\sup \left\{|\langle a, b\rangle| \mid \begin{array}{l}
a \in A \cap(A \cap B)^{\perp},\|a\| \leq 1, \\
b \in B \cap(A \cap B)^{\perp},\|b\| \leq 1
\end{array}\right\} .
\end{aligned}
$$

Let us gather some properties of angles.

Fact 7.10 Let $A$ and $B$ be linear subspaces of $X$. Then the following hold:

(i) If $A \cap B=\{0\}$, then $c(A, B)=c_{0}(A, B)$.

(ii) If $A \cap B \neq\{0\}$, then $c_{0}(A, B)=1$.

(iii) $c(A, B)<1$.

(iv) $c(A, B)=c_{0}\left(A, B \cap(A \cap B)^{\perp}\right)=c_{0}\left(A \cap(A \cap B)^{\perp}, B\right)$.

(v) (Solmon) $c(A, B)=c\left(A^{\perp}, B^{\perp}\right)$. 
Proof. (i)-(iii): Clear from the definitions. (iv): See, e.g., [8, Lemma 2.10(1)] or [9, Lemma 9.5]. (v): See, e.g., [8, Theorem 2.16].

Proposition 7.11 (CQ-number of two linear subspaces and Dixmier angle) Let $A$ and $B$ be linear subspaces of $X$, and let $\delta>0$. Then

$$
\begin{aligned}
& \theta_{\delta}(A, A, B, B)=c_{0}\left(A^{\perp} \cap(A+B), B^{\perp} \cap(A+B)\right), \\
& \theta_{\delta}(A, X, B, B)=c_{0}\left(A^{\perp} \cap(A+B), B^{\perp}\right), \\
& \theta_{\delta}(A, A, B, X)=c_{0}\left(A^{\perp}, B^{\perp} \cap(A+B)\right),
\end{aligned}
$$

where the CQ-numbers at 0 are defined as in (94).

Proof. This follows from Proposition 7.8.

We are now in a position to derive a striking connection between the CQ-number and the Friedrichs angle, which underlines a possible interpretation of the CQ-number as a generalized Friedrichs angle between two sets.

Theorem 7.12 (CQ-number of two linear subspaces and Friedrichs angle) Let $A$ and $B$ be linear subspaces of $X$, and let $\delta>0$. Then

$$
\theta_{\delta}(A, A, B, B)=\theta_{\delta}(A, X, B, B)=\theta_{\delta}(A, A, B, X)=c(A, B)<1,
$$

where the CQ-number at 0 is defined as in (94).

Proof. On the one hand, using Fact 7.10(v), we have

$$
\begin{aligned}
c(A, B) & =c\left(A^{\perp}, B^{\perp}\right) \\
& =c_{0}\left(A^{\perp} \cap\left(A^{\perp} \cap B^{\perp}\right)^{\perp}, B^{\perp} \cap\left(A^{\perp} \cap B^{\perp}\right)^{\perp}\right) \\
& =c_{0}\left(A^{\perp} \cap(A+B), B^{\perp} \cap(A+B)\right) .
\end{aligned}
$$

On the other hand, Fact 7.10(iv) yields

$$
\begin{aligned}
c_{0}\left(A^{\perp} \cap(A+B), B^{\perp}\right) & =c_{0}\left(A^{\perp} \cap\left(A^{\perp} \cap B^{\perp}\right)^{\perp}, B^{\perp}\right) \\
& =c\left(A^{\perp}, B^{\perp}\right) \\
& =c_{0}\left(A^{\perp}, B^{\perp} \cap\left(A^{\perp} \cap B^{\perp}\right)^{\perp}\right) \\
& =c_{0}\left(A^{\perp}, B^{\perp} \cap(A+B)\right) .
\end{aligned}
$$

Altogether, recalling Proposition 7.11, we obtain the result.

The results in this subsection have a simple generalization to intersecting affine subspaces. Indeed, if $A$ and $B$ are intersecting affine subspaces, then the corresponding Friedrichs angle is

$$
c(A, B):=c(\operatorname{par} A, \operatorname{par} B) .
$$

Combining (99) with Theorem 7.12, we immediately obtain the following result. 
Corollary 7.13 (CQ-number of two intersecting affine subspaces and Friedrichs angle) Let $A$ and $B$ be affine subspaces of $X$, suppose that $c \in A \cap B$, and let $\delta>0$. Then

$$
\theta_{\delta}(A, A, B, B)=\theta_{\delta}(A, X, B, B)=\theta_{\delta}(A, A, B, X)=c(A, B)<1,
$$

where the CQ-number at $c$ is defined as in (94).

\section{Regularities}

In this section, we study a notion of set regularity that is based on restricted normal cones.

Definition 8.1 (regularity and superregularity) Let $A$ and $B$ be nonempty subsets of $X$, and let $c \in X$.

(i) We say that $B$ is $(A, \varepsilon, \delta)$-regular at $c \in X$ if $\varepsilon \geq 0, \delta>0$, and

$$
\left.\begin{array}{c}
(y, b) \in B \times B, \\
\|y-c\| \leq \delta,\|b-c\| \leq \delta, \\
u \in \widehat{N}_{B}^{A}(b)
\end{array}\right\} \Rightarrow\langle u, y-b\rangle \leq \varepsilon\|u\| \cdot\|y-b\| .
$$

If $B$ is $(X, \varepsilon, \delta)$-regular at $c$, then we also simply speak of $(\varepsilon, \delta)$-regularity.

(ii) The set $B$ is called $A$-superregular at $c \in X$ if for every $\varepsilon>0$ there exists $\delta>0$ such that $B$ is $(A, \varepsilon, \delta)$-regular at $c$. Again, if $B$ is $X$-superregular at $c$, then we also say that $B$ is superregular at $c$.

Remark 8.2 Several comments on Definition 8.1 are in order.

(i) Superregularity with $A=X$ was introduced by Lewis, Luke and Malick in [12, Section 4]. Among other things, they point out that amenability and prox regularity are sufficient conditions for superregularity, while Clarke regularity is a necessary condition. Moreover, an important subclass of prox regular sets are $C^{2}$ manifolds [13].

(ii) The reference point $c$ does not have to belong to $B$. If $c \notin \bar{B}$, then for every $\delta \in] 0, d_{B}(c)[, B$ is $(0, \delta)$-regular at $c$; consequently, $B$ is superregular at $c$.

(iii) If $\varepsilon \in[1,+\infty[$, then Cauchy-Schwarz implies that $B$ is $(\varepsilon,+\infty)$-regular at every point in $X$.

(iv) It follows from Proposition 2.7(ii) that $B$ is $\left(A_{1} \cup A_{2}, \varepsilon, \delta\right)$-regular at $c$ if and only if $B$ is both $\left(A_{1}, \varepsilon, \delta\right)$-regular and $\left(A_{2}, \varepsilon, \delta\right)$-regular at $c$.

(v) If $B$ is convex, then it follows with Lemma 2.4(vii) that $B$ is $(A, 0,+\infty)$-regular at $c$; consequently, $B$ is superregular.

(vi) Similarly, if $B$ is locally convex at $c$, i.e., there exists $\rho \in \mathbb{R}_{++}$such that $B \cap$ ball $(c ; \rho)$ is convex, then $B$ is superregular at $c$. 
(vii) If $B$ is $(A, 0, \delta)$-regular at $c$, then $B$ is $A$-superregular at $c$; the converse, however, is not true in general (see Example 8.3 below).

As a first example, let us consider the sphere, which is a $C^{2}$ manifold and which allows us to explicitly quantify regularity.

Example 8.3 (sphere) Let $z \in X$ and $\rho \in \mathbb{R}_{++}$. Set $S:=\operatorname{sphere}(z ; \rho)$, suppose that $s \in S$, let $\varepsilon \in \mathbb{R}_{++}$, and let $\delta \in \mathbb{R}_{++}$. Then $S$ is $(\varepsilon, \rho \varepsilon)$-regular at $s$; consequently, $S$ is superregular at $s$ (see Definition 8.1). However, $S$ is not $(0, \delta)$-regular at $s$.

Proof. Let $b \in S$ and $y \in S$. Then $\rho^{2}=\|z-y\|^{2}=\|z-b\|^{2}+\|y-b\|^{2}-2\langle z-b, y-b\rangle=$ $\rho^{2}+\|y-b\|^{2}-2\langle z-b, y-b\rangle$, which implies

$$
2\langle z-b, y-b\rangle=\|y-b\|^{2} .
$$

On the other hand, by Example 2.6, we have

$$
\widehat{N}_{S}^{X}(b) \cap \operatorname{sphere}(0 ; 1)=\left\{ \pm \frac{z-b}{\|z-b\|}\right\}=\left\{ \pm \frac{z-b}{\rho}\right\} .
$$

Suppose that $u \in \widehat{N}_{S}^{X}(b) \cap$ sphere $(0 ; 1)$. Combining (147) and (148), we obtain

$$
\left\langle\widehat{N}_{S}^{X}(b) \cap \operatorname{sphere}(0 ; 1), y-b\right\rangle=\left\{ \pm \frac{1}{2 \rho}\|y-b\|^{2}\right\} \text {. }
$$

Thus if $\|y-s\| \leq \rho \varepsilon,\|b-s\| \leq \rho \varepsilon$, and $u \in \widehat{N}_{S}^{X}(b) \cap$ sphere $(0 ; 1)$, then

$$
\begin{aligned}
\langle u, y-b\rangle & \leq \frac{1}{2 \rho}\|y-b\|^{2} \leq \frac{1}{2 \rho}(\|y-s\|+\|s-b\|)\|y-b\| \leq \frac{\rho \varepsilon+\rho \varepsilon}{2 \rho}\|y-b\| \\
& =\varepsilon\|u\| \cdot\|y-b\|,
\end{aligned}
$$

which verifies the $(\varepsilon, \rho \varepsilon)$-regularity of $S$ at $s$. Finally, by (149),

$$
\max \left\{\left\langle\widehat{N}_{S}^{X}(b) \cap \operatorname{sphere}(0 ; 1), y-b\right\rangle\right\}=\frac{1}{2 \rho}\|y-b\|^{2}>0
$$

and therefore $S$ is not $(0, \delta)$-regular at $s$.

We now characterizes $A$-superregularity using restricted normal cones.

Theorem 8.4 (characterization of $A$-superregularity) Let $A$ and $B$ be nonempty subsets of $X$, and let $c \in X$. Then $B$ is $A$-superregular at $c$ if and only if for every $\varepsilon \in \mathbb{R}_{++}$, there exists $\delta \in \mathbb{R}_{++}$such that

$$
\left.\begin{array}{c}
(y, b) \in B \times B \\
\|y-c\| \leq \delta,\|b-c\| \leq \delta \\
u \in N_{B}^{A}(b)
\end{array}\right\} \Rightarrow\langle u, y-b\rangle \leq \varepsilon\|u\| \cdot\|y-b\| .
$$


Proof. " $\Leftarrow$ ": Clear from Lemma 2.4(iv). " $\Rightarrow$ ": We argue by contradiction; thus, we assume there exists $\varepsilon \in \mathbb{R}_{++}$and sequences $\left(y_{n}, b_{n}, u_{n}\right)_{n \in \mathbb{N}}$ in $B \times B \times X$ such that $\left(y_{n}, b_{n}\right) \rightarrow(c, c)$ and for every $n \in \mathbb{N}$,

$$
u_{n} \in N_{B}^{A}\left(b_{n}\right) \quad \text { and } \quad\left\langle u_{n}, y_{n}-b_{n}\right\rangle>\varepsilon\left\|u_{n}\right\| \cdot\left\|y_{n}-b_{n}\right\| .
$$

By the definition of the restricted normal cone, for every $n \in \mathbb{N}$, there exists a sequence $\left(b_{n, k}, u_{n, k}\right)_{k \in \mathbb{N}}$ in $B \times X$ such that $\lim _{k \in \mathbb{N}} b_{n, k}=b_{n}, \lim _{k \in \mathbb{N}} u_{n, k}=u_{n}$, and $(\forall k \in \mathbb{N}) u_{n, k} \in \widehat{N}_{B}^{A}\left(b_{n, k}\right)$. Hence there exists a subsequence $\left(k_{n}\right)_{n \in \mathbb{N}}$ of $(n)_{n \in \mathbb{N}}$ such that $b_{n, k_{n}} \rightarrow c$ and

$$
(\forall n \in \mathbb{N}) \quad\left\langle u_{n, k_{n}}, y_{n}-b_{n, k_{n}}\right\rangle>\frac{\varepsilon}{2}\left\|u_{n, k_{n}}\right\| \cdot\left\|y_{n}-b_{n, k_{n}}\right\| .
$$

However, this contradicts the $A$-superregularity of $B$ at $c$.

When $B=X$, then Theorem 8.4 turns into [12, Proposition 4.4]:

Corollary 8.5 (Lewis-Luke-Malick) Let $B$ be a nonempty subset of $X$ and let $c \in B$. Then $B$ is superregular at $c$ if and only if for every $\varepsilon \in \mathbb{R}_{++}$there exists $\delta \in \mathbb{R}_{++}$such that

$$
\left.\begin{array}{c}
(y, b) \in B \times B \\
\|y-c\| \leq \delta,\|b-c\| \leq \delta \\
u \in N_{B}(b)
\end{array}\right\} \Rightarrow\langle u, y-b\rangle \leq \varepsilon\|u\| \cdot\|y-b\| .
$$

We now introduce the notion of joint-regularity, which is tailored for collections of sets and which turns into Definition 8.1 when the index set is a singleton.

Definition 8.6 (joint-regularity) Let $A$ be a nonempty subset of $X$, let $\mathcal{B}:=\left(B_{j}\right)_{j \in J}$ be a nontrivial collection of nonempty subsets of $X$, and let $c \in X$.

(i) We say that $\mathcal{B}$ is $(A, \varepsilon, \delta)$-joint-regular at $c$ if $\varepsilon \geq 0, \delta>0$, and for every $j \in J, B_{j}$ is $(A, \varepsilon, \delta)$-regular at $c$.

(ii) The collection $\mathcal{B}$ is $A$-joint-superregular at $c$ if for every $j \in J, B_{j}$ is $A$-superregular at $c$.

As in Definition 8.1, we may omit the prefix $A$ if $A=X$.

Here are some verifiable conditions that guarantee joint-(super)regularity.

Proposition 8.7 Let $\mathcal{A}:=\left(A_{j}\right)_{j \in J}$ and $\mathcal{B}:=\left(B_{j}\right)_{j \in J}$ be nontrivial collections of nonempty subsets of $X$, let $c \in X$, let $\left(\varepsilon_{j}\right)_{j \in J}$ be a collection in $\mathbb{R}_{+}$, and let $\left(\delta_{j}\right)_{j \in J}$ be a collection in $\left.] 0,+\infty\right]$. Set $A:=\bigcap_{j \in J} A_{j}$, $\varepsilon:=\sup _{j \in J} \varepsilon_{j}$, and $\delta:=\inf _{j \in J} \delta_{j}$. Then the following hold:

(i) If $\delta>0$ and $(\forall j \in J) B_{j}$ is $\left(A_{j}, \varepsilon_{j}, \delta_{j}\right)$-regular at $c$, then $\mathcal{B}$ is $(A, \varepsilon, \delta)$-joint-regular at $c$.

(ii) If $J$ is finite and $(\forall j \in J) B_{j}$ is $\left(A_{j}, \varepsilon_{j}, \delta_{j}\right)$-regular at $c$, then $\mathcal{B}$ is $(A, \varepsilon, \delta)$-joint-regular at $c$. 
(iii) If $J$ is finite and $(\forall j \in J) B_{j}$ is $A_{j}$-superregular at $c$, then $\mathcal{B}$ is $A$-joint-superregular at $c$.

Proof. (i): Indeed, by Remark 8.2(iv), $B_{j}$ is $(A, \varepsilon, \delta)$-regular at $c$ for every $j \in J$.

(ii): Since $J$ is finite, we have $\delta>0$ and so the conclusion follows from (i).

(iii): This follows from (ii) and the definitions.

Corollary 8.8 (convexity and regularity) Let $\mathcal{B}:=\left(B_{j}\right)_{j \in J}$ be a nontrivial collection of nonempty convex subsets of $X$, let $A \subseteq X$, and let $c \in X$. Then $\mathcal{B}$ is $(0,+\infty)$-joint-regular, $(A, 0,+\infty)$-joint-regular, joint-superregular, and $A$-joint-superregular at c.

Proof. By Remark $8.2(\mathrm{v}), B_{j}$ is $(0,+\infty)$-regular, superregular, and $A$-superregular at $c$, for every $j \in J$. Now apply Proposition 8.7(i)\&(iii).

The following example illustrates the flexibility gained through the notion of joint-regularity.

Example 8.9 (two lines: joint-superregularity $\nRightarrow$ superregularity of the union) Suppose that $d_{1}$ and $d_{2}$ are in sphere $(0 ; 1)$. Set $B_{1}:=\mathbb{R} d_{1}, B_{2}:=\mathbb{R} d_{2}$, and $B:=B_{1} \cup B_{2}$, and assume that $B_{1} \cap B_{2}=$ $\{0\}$. By Corollary 8.8, $\left(B_{1}, B_{2}\right)$ is joint-superregular at 0 . Let $\delta \in \mathbb{R}_{++}$, and set $b:=\delta d_{1}$ and $y:=\delta d_{2}$. Then $\|y-0\|=\delta,\|b-0\|=\delta$, and $0<\|y-b\|=\delta\left\|d_{2}-d_{1}\right\|$. Using Proposition 2.3(iii), we see that $N_{B}(b)=\left\{d_{1}\right\}^{\perp}$. Note that there exists $v \in\left\{d_{1}\right\}^{\perp}$ such that $\left\langle v, d_{2}\right\rangle \neq 0$ (for otherwise $\left\{d_{1}\right\}^{\perp} \subseteq\left\{d_{2}\right\}^{\perp} \Rightarrow B_{2} \subseteq B_{1}$, which is absurd). Hence there exists $u \in\left\{d_{1}\right\}^{\perp}=\{b\}^{\perp}=N_{B}(b)$ such that $\|u\|=1$ and $\left\langle u, d_{2}\right\rangle>0$. It follows that $\langle u, y-b\rangle=\langle u, y\rangle=\delta\left\langle u, d_{2}\right\rangle=\left\langle u, d_{2}\right\rangle\|u\| \| y-$ $b\|/\| d_{2}-d_{1} \|$. Therefore, $B$ is not superregular at 0 .

Let us provide an example of an $A$-superregular set that is not superregular. To do so, we require the following elementary result.

Lemma 8.10 Consider in $\mathbb{R}^{2}$ the sets $C:=\left[(0,1),\left(m, 1+m^{2}\right)\right]=\{(x, 1+m x) \mid x \in[0, m]\}$ and $D:=\left[(m, 1),\left(m, 1+m^{2}\right)\right]$, where $m \in \mathbb{R}_{++}$. Let $z \in \mathbb{R}$. Then

$$
P_{C \cup D}(z, 0)= \begin{cases}(0,1), & \text { if } z<m / 2 \\ \{(0,1),(m, 1)\}, & \text { if } z=m / 2 \\ (m, 1), & \text { if } z>m / 2\end{cases}
$$

Proof. It is clear that $P_{D}(z, 0)=(m, 1)$. We assume that $0<z<m$ for otherwise (157) is clearly true. We claim that $P_{C}(z, 0)=(0,1)$. Indeed, $f: x \mapsto\|(x, 1+m x)-(z, 0)\|^{2}$ is a convex quadratic with minimizer $x_{z}:=(z-m) /\left(1+m^{2}\right)$. The requirement $x_{z} \geq 0$ from the definition of $C$ forces $z \geq m$, which is a contradiction. Hence $P_{C}(z, 0)$ is a subset of the relative boundary of $C$, i.e., of $\left\{(0,1),\left(m, 1+m^{2}\right)\right\}$. Clearly, $(0,1)$ is the closer to $(z, 0)$ than $\left(m, 1+m^{2}\right)$. This verifies the claim. Since $P_{C \cup D}(z, 0)$ is the subset of points in $P_{C}(z, 0) \cup P_{D}(z, 0)$ closest to $(z, 0)$, the result follows. 
Example 8.11 ( $A$-superregularity $\not \Rightarrow$ superregularity) Suppose that $X=\mathbb{R}^{2}$. As in $[12$, Example 4.6], we consider $c:=(0,0) \in X$ and $B:=\operatorname{epi} f$, where

$$
f: \mathbb{R} \rightarrow]-\infty,+\infty]: x \mapsto \begin{cases}2^{k}\left(x-2^{k}\right), & \text { if } 2^{k} \leq x<2^{k+1} \text { and } k \in \mathbb{Z} ; \\ 0, & \text { if } x=0 ; \\ +\infty, & \text { if } x<0 .\end{cases}
$$

Then $B$ is not superregular at $c$; however, $B$ is $A$-superregular at $c$, where $A:=\mathbb{R} \times\{-1\}$.

Proof. It is stated in [12, Example 4.6] that $B$ is not superregular at $c$ (and that $B$ is Clarke regular at $c$ ).

To tackle $A$-superregularity, let us determine $P_{B}(A)$. Let us consider the point $a=(\alpha,-1)$, where $\alpha \in\left[2^{-1}, 1[\right.$. Then Lemma 8.10 (see also the picture below) implies that

$$
P_{B}(\alpha,-1)= \begin{cases}\left(\frac{1}{2}, 0\right), & \text { if } \frac{1}{2} \leq \alpha<\frac{3}{4} \\ \left\{\left(\frac{1}{2}, 0\right),(1,0)\right\}, & \text { if } \alpha=\frac{3}{4} \\ (1,0), & \text { if } \frac{3}{4}<\alpha<1\end{cases}
$$

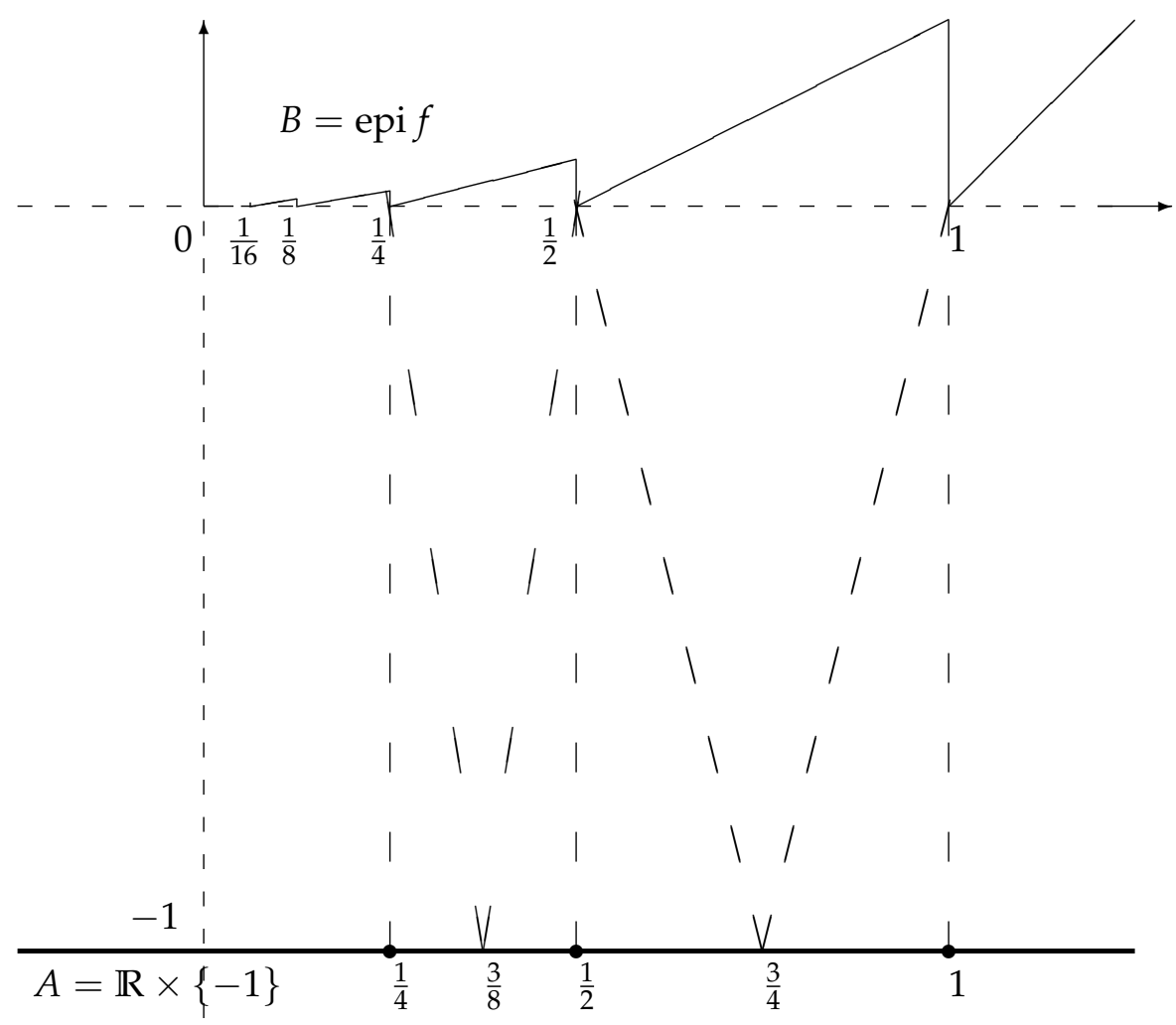


and more generally,

$$
2^{k} \leq \alpha<2^{k+1} \Rightarrow P_{B}(\alpha,-1)= \begin{cases}\left(2^{k}, 0\right), & \text { if } 2^{k} \leq \alpha<2^{k}+2^{k-1} \\ \left\{\left(2^{k}, 0\right),\left(2^{k+1}, 0\right)\right\}, & \text { if } \alpha=2^{k}+2^{k-1} \\ \left(2^{k+1}, 0\right), & \text { if } 2^{k}+2^{k-1}<\alpha<2^{k+1}\end{cases}
$$

Clearly, if $a \in \mathbb{R}_{-} \times\{-1\}$, then $P_{B}(a)=(0,0)$. Let $b \in B$. Then

$$
A \cap P_{B}^{-1}(b)= \begin{cases}{\left[2^{k-2}+2^{k-1}, 2^{k-1}+2^{k}\right] \times\{-1\},} & \text { if } b=\left(2^{k}, 0\right) \text { and } k \in \mathbb{Z} ; \\ \mathbb{R}_{-} \times\{-1\}, & \text { if } b=(0,0) ; \\ \varnothing, & \text { otherwise. }\end{cases}
$$

Thus

$$
\widehat{N}_{B}^{A}(b)= \begin{cases}\operatorname{cone}\left(\left[-2^{k-2}, 2^{k-1}\right] \times\{-1\}\right), & \text { if } b=\left(2^{k}, 0\right) \text { and } k \in \mathbb{Z} ; \\ \{(0,0)\} \cup\left(\mathbb{R}_{-} \times \mathbb{R}_{--}\right), & \text {if } b=(0,0) ; \\ \{(0,0)\}, & \text { otherwise. }\end{cases}
$$

Let $\varepsilon \in \mathbb{R}_{++}$. Let $K \in \mathbb{Z}$ be such that $2^{K-1} \leq \varepsilon$, and let $\left.\left.\delta \in\right] 0,2^{K}\right]$. Furthermore, let $y=\left(y_{1}, y_{2}\right) \in$ $B$, let $b=\left(b_{1}, b_{2}\right) \in B$, let $u \in \widehat{N}_{B}^{A}(b)$, and assume that $\|y-c\| \leq \delta$ and that $\|b-c\| \leq \delta$. We consider three cases.

Case 1: $b=(0,0)$. Then $u \in \mathbb{R}_{-}^{2}$ and $y \in \mathbb{R}_{+}^{2} ;$ consequently, $\langle u, y-b\rangle=\langle u, y\rangle \leq 0 \leq \varepsilon\|u\| \cdot \| y-$ $b \|$.

Case 2: $b \notin\left(\{0\} \cup 2^{\mathbb{Z}}\right) \times\{0\}$. Then $\widehat{N}_{B}^{A}(b)=\{(0,0\}$; hence $u=0$ and so $\langle u, y-b\rangle=0 \leq$ $\varepsilon\|u\| \cdot\|y-b\|$.

Case 3: $b \in 2^{\mathbb{Z}} \times\{0\}$, say $b=\left(2^{k}, 0\right)$, where $k \in \mathbb{Z}$. Since $2^{k}=\|b-0\|=\|b-c\| \leq \delta \leq 2^{K}$, we have $k \leq K$. Furthermore, $y_{2} \geq 0, \max \left\{\left|y_{1}-b_{1}\right|,\left|y_{2}-b_{2}\right|\right\} \leq\|y-b\|$, and $u=\lambda(t,-1)=$ $(\lambda t,-\lambda)$ where $t \in\left[-2^{k-2}, 2^{k-1}\right]$ and $\lambda \geq 0$. Hence $\lambda \leq\|u\|$ and

$$
\begin{aligned}
\langle u, y-b\rangle & =\lambda t\left(y_{1}-b_{1}\right)-\lambda\left(y_{2}-b_{2}\right)=\lambda t\left(y_{1}-b_{1}\right)-\lambda\left(y_{2}-0\right) \\
& \leq \lambda t\left(y_{1}-b_{1}\right) \leq \lambda|t| \cdot\left|y_{1}-b\right| \\
& \leq\|u\| \cdot 2^{k-1} \cdot\|y-b\| \leq 2^{K-1}\|u\| \cdot\|y-b\| \leq \varepsilon \cdot\|u\| \cdot\|y-b\| .
\end{aligned}
$$

Therefore, in all three cases, we have shown that $\langle u, y-b\rangle \leq \varepsilon\|u\| \cdot\|y-b\|$.

Finally, we use Example 8.11 to construct an example complementary to Example 8.9.

Example 8.12 (superregularity of the union $\nRightarrow$ joint-superregularity) Suppose that $X=\mathbb{R}^{2}$, set $B_{1}:=$ epi $f$, where $f$ is as in Example 8.11, $B_{2}:=X \backslash B_{1}$, and $c:=(0,0)$. Since $B_{1} \cup B_{2}=X$ is convex, it is clear from Remark 8.2(v) that $B_{1} \cup B_{2}$ is superregular at $c$. On the other hand, since $B_{1}$ is not superregular at $c$ (see Example 8.11), it is obvious that $\left(B_{1}, B_{2}\right)$ is not joint-superregular at $c$. 


\section{Acknowledgments}

We would like to thank the referee for her/his helpful comments. HHB was partially supported by the Natural Sciences and Engineering Research Council of Canada and by the Canada Research Chair Program. This research was initiated when HHB visited the Institut für Numerische und Angewandte Mathematik, Universität Göttingen because of his study leave in Summer 2011. HHB thanks DRL and the Institut for their hospitality. DRL was supported in part by the German Research Foundation grant SFB755-A4. HMP was partially supported by the Pacific Institute for the Mathematical Sciences and and by a University of British Columbia research grant. XW was partially supported by the Natural Sciences and Engineering Research Council of Canada.

\section{References}

[1] H.H. Bauschke, J.M. Borwein, and A.S. Lewis, The method of cyclic projections for closed convex sets in Hilbert space, in Recent Developments in Optimization Theory and Nonlinear Analysis (Jerusalem 1995), Y. Censor and S. Reich (editors), Contemporary Mathematics vol. 204, American Mathematical Society, pp. 1-38, 1997.

[2] H.H. Bauschke and P.L. Combettes, Convex Analysis and Monotone Operator Theory in Hilbert Spaces, Springer, 2011.

[3] H.H. Bauschke, D.R. Luke, H.M. Phan, and X. Wang, Restricted normal cones and sparsity optimization with affine constraints, arXiv preprint, May 2012, http://arxiv.org

[4] H.H. Bauschke, D.R. Luke, H.M. Phan, and X. Wang, Restricted normal cones and the method of alternating projections: applications, preprint, March 2013.

[5] J.M. Borwein and Q.J. Zhu, Techniques of Variational Analysis, Springer-Verlag, 2005.

[6] Y. Censor and S.A. Zenios, Parallel Optimization, Oxford University Press, 1997.

[7] F.H. Clarke, Y.S. Ledyaev, R.J. Stern and P.R. Wolenski, Nonsmooth Analysis and Control Theory, Springer-Verlag, 1998.

[8] F. Deutsch, The angle between subspaces of a Hilbert space, in Approximation theory, wavelets and applications (Maratea, 1994), S.P. Singh, A. Carbone, and B. Watson (editors), NATO Advanced Science Institutes Series C: Mathematical and Physical Sciences vol. 454, Kluwer, pp. 107-130, 1995.

[9] F. Deutsch, Best Approximation in Inner Product Spaces, Springer, 2001.

[10] J. Dixmier, Étude sur les variétés et les opérateurs de Julia, avec quelques applications, Bulletin de la Société Mathématique de France 77 (1949), 11-101.

[11] K. Friedrichs, On certain inequalities and characteristic value problems for analytic functions and for functions of two variables, Transactions of the AMS 41 (1937), 321-364. 
[12] A.S. Lewis, D.R. Luke, and J. Malick, Local linear convergence for alternating and averaged nonconvex projections, Foundations of Computational Mathematics 9 (2009), 485-513.

[13] A.S. Lewis and J. Malick, Alternating projection on manifolds, Mathematics of Operations Research 33 (2008), 216-234.

[14] P.D. Loewen, Optimal Control via Nonsmooth Analysis, CRM Proceedings \& Lecture Notes, AMS, Providence, RI, 1993.

[15] B.S. Mordukhovich, Variational Analysis and Generalized Differentiation I, Springer-Verlag, 2006.

[16] R.T. Rockafellar, Convex Analysis, Princeton University Press, Princeton, 1970.

[17] R.T. Rockafellar and R.J-B Wets, Variational Analysis, Springer, corrected 3rd printing, 2009.

[18] J. von Neumann, Functional Operators Vol.II. The Geometry of Orthogonal Spaces, Annals of Mathematical Studies \#22, Princeton University Press, Princeton, 1950.

[19] N. Wiener, On the factorization of matrices, Commentarii Mathematici Helvetici 29 (1955), 97111.

[20] C. Zălinescu, Convex Analysis in General Vector Spaces, World Scientific Publishing, 2002. 